Nonl i near stabi lity of magnet i c i sl ands in a rot at ing hel i cal pl asma

\begin{tabular}{|c|c|}
\hline $\begin{array}{l}\text { jour nal or } \\
\text { publ i cat } i \text { on } \mathrm{title}\end{array}$ & Physi cs of PI astas \\
\hline vol une & Vol . 19 \\
\hline nunber & I ssue 12 \\
\hline page $r$ ange & $122510-1-122510-15$ \\
\hline year & 2012-12-01 \\
\hline URL & ht t p: //hdl . handl e. net /10655/8991 \\
\hline
\end{tabular}




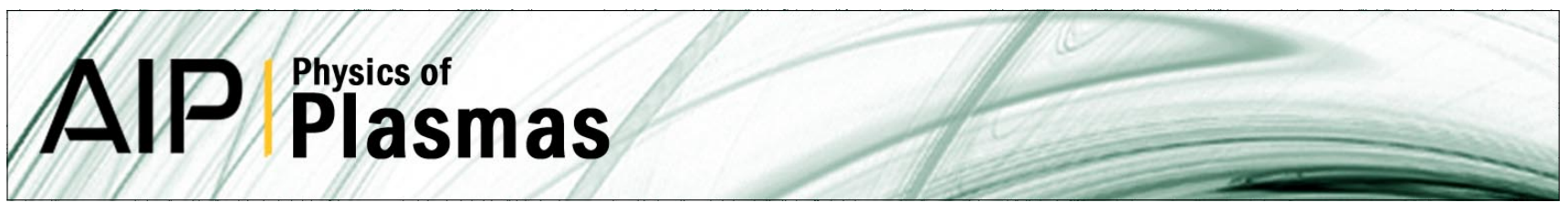

\section{Nonlinear stability of magnetic islands in a rotating helical plasma}

S. Nishimura, S. Toda, M. Yagi, and Y. Narushima

Citation: Phys. Plasmas 19, 122510 (2012); doi: 10.1063/1.4773041

View online: http://dx.doi.org/10.1063/1.4773041

View Table of Contents: http://pop.aip.org/resource/1/PHPAEN/v19/i12

Published by the American Institute of Physics.

\section{Related Articles}

Nonlinear dynamics of beta-induced Alfvén eigenmode in tokamak

Phys. Plasmas 20, 012510 (2013)

Linear and nonlinear wave propagation in weakly relativistic quantum plasmas

Phys. Plasmas 20, 012114 (2013)

Time-domain simulation of nonlinear radiofrequency phenomena

Phys. Plasmas 20, 012116 (2013)

Small amplitude nonlinear electron acoustic solitary waves in weakly magnetized plasma

Phys. Plasmas 20, 012113 (2013)

Dust-acoustic shock formation in dusty plasmas with non-thermal ions

Phys. Plasmas 20, 013704 (2013)

\section{Additional information on Phys. Plasmas}

Journal Homepage: http://pop.aip.org/

Journal Information: http://pop.aip.org/about/about_the_journal

Top downloads: http://pop.aip.org/features/most_downloaded

Information for Authors: http://pop.aip.org/authors

\section{ADVERTISEMENT}

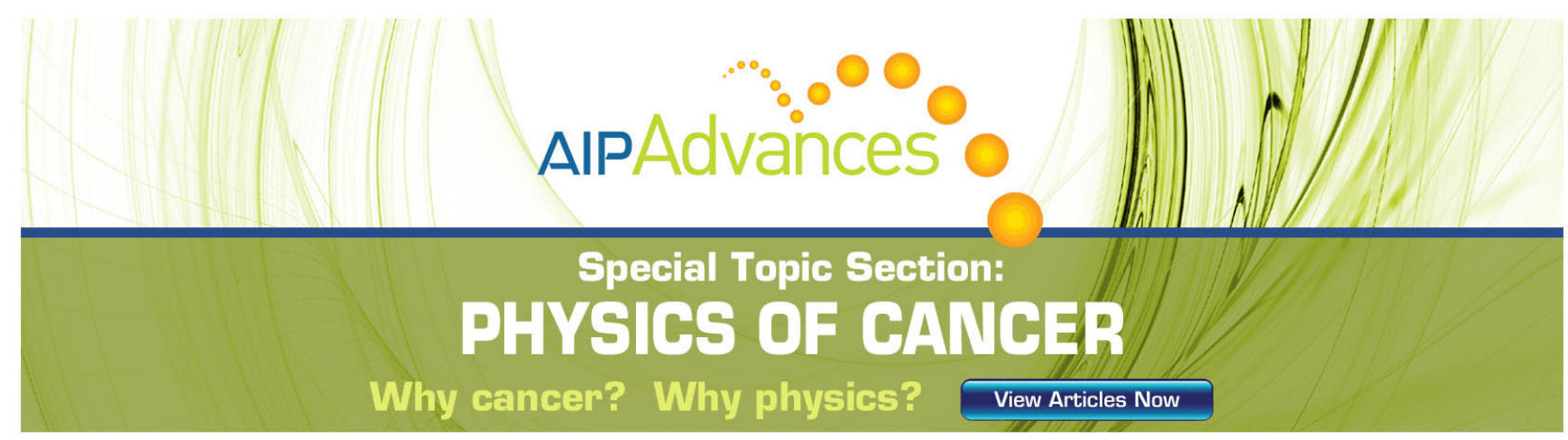




\title{
Nonlinear stability of magnetic islands in a rotating helical plasma
}

\author{
S. Nishimura, ${ }^{1}$ S. Toda,${ }^{1}$ M. Yagi, ${ }^{2}$ and Y. Narushima ${ }^{1}$ \\ ${ }^{1}$ National Institute for Fusion Science, Toki, Gifu 509-5292, Japan \\ ${ }^{2}$ Japan Atomic Energy Agency, Rokkasho, Aomori 039-3212, Japan
}

(Received 15 October 2012; accepted 5 December 2012; published online 28 December 2012)

\begin{abstract}
Coexistence of the forced magnetic reconnection by a resonant magnetic perturbation (RMP) and the curvature-driven tearing mode is investigated in a helical (stellarator) plasma rotated by helical trapped particle-induced neoclassical flows. A set of Rutherford-type equations of rotating magnetic islands and a poloidal flow evolution equation is revisited. Using the model, analytical expressions of criteria of spontaneous shrinkage (self-healing) of magnetic islands and sudden growth of locked magnetic islands (penetration of RMP) are obtained, where nonlinear saturation states of islands show bifurcation structures and hysteresis characteristics. Considering radial profile of poloidal flows across magnetic islands, it is found that the self-healing is driven by neoclassical viscosity even in the absence of micro-turbulence-induced anomalous viscosity. Effects of unfavorable curvature in stellarators are found to modify the critical values. The scalings of criteria are consistent with low- $\beta$ experiments in the large helical device. (C) 2012 American Institute of Physics. [http://dx.doi.org/10.1063/1.4773041]
\end{abstract}

\section{INTRODUCTION}

In magnetic confinement fusion with toroidal devices, such as tokamaks and stellarators, nested magnetic surfaces are often broken by the magnetic reconnection, and consequent magnetic island structure strongly affects plasma confinement property. ${ }^{1}$ Instability which excites magnetic islands is called the tearing mode. ${ }^{2-5}$ Even if the intrinsic tearing mode is stable, magnetic islands are produced by external resonant magnetic perturbation (RMP) through the mechanism of the forced magnetic reconnection. ${ }^{6}$ In tokamaks, much theoretical work has been done for influence of plasma flows on stability of RMP-driven magnetic islands. $^{7-17}$ An essential point is that island growth, in other words, penetration of RMP, is triggered when plasma flows are locked by RMP, otherwise, islands are damped.

In the large helical device (LHD) ${ }^{18-22}$ and the TJ-II, ${ }^{23}$ spontaneous shrinkage of RMP-driven magnetic islands has been observed, known as the self-healing. It has been pointed out that the perturbed bootstrap current, the polarization current, and the curvature-driven current are hopeless to explain the self-healing mechanism in low- $\beta$ regime, ${ }^{18,20,21}$ where $\beta$ is a ratio of the total plasma pressure and the magnetic pressure. For this reason, by analogy with tokamaks, it has been attempted to explain the self-healing mechanism by the screening effect of helical trapped particle-induced neoclassical flows. ${ }^{24-29}$

However, a problematic point is that, historically, the magnetic reconnection in stellarators (helical systems) has been investigated in the context of the curvature-driven tearing mode. The curvature-driven current perturbation, such as the resonant Pfirsch-Schlüter current, is excited by normal magnetic field line curvature, and magnetic islands are generated to sustain three-dimensional magnetohydrodynamics (MHD) equilibria. ${ }^{30-34}$ In particular, the curvature-driven tearing mode is crucial in high- $\beta$ regime. In addition, effective curvature is easily controlled in stellarators. For this reason, comprehensive understanding of the RMP-driven mode and the curvature-driven mode is necessary to know possible saturation states of magnetic islands, where theoretical modeling is an open issue.

In this paper, we revisit the theoretical model of the magnetic reconnection in a helical plasma, so that RMP, plasma flows, and the curvature-driven tearing mode are described simultaneously. The paper is organized as follows. In Sec. II, we introduce basic fluid models with neoclassical viscosity in a stellarator. In Sec. III, we derive model equations for magnetic islands and poloidal flows. In Sec. IV, the model is analytically solved, and the self-healing and penetration thresholds and scalings of them are derived. In Sec. V, the model is numerically solved in a typical parameter regime in LHD. Sections VI and VII are devoted for discussion and summary.

\section{BASIC MODEL}

\section{A. Two-fluid model with neoclassical viscosity}

An extended version of the two fluid model ${ }^{35,36}$ for hydrogen plasmas with the Braginskii's classical transport closure $^{37}$ and additional neoclassical viscosity in a torus magnetic field is written as

$$
\begin{gathered}
\frac{d_{\mathrm{i}} n_{\mathrm{i}}}{d t}+n_{\mathrm{i}} \nabla \cdot \boldsymbol{V}_{\mathrm{i}}=0, \\
\frac{d_{\mathrm{e}} n_{\mathrm{e}}}{d t}+n_{\mathrm{e}} \nabla \cdot \boldsymbol{V}_{\mathrm{e}}=0 \\
m_{\mathrm{i}} n_{\mathrm{i}}\left(\frac{d_{\mathrm{i}} \boldsymbol{V}_{E}}{d t}+\frac{d \boldsymbol{V}_{\|}}{d t}\right)=-\nabla p+\frac{1}{c} \boldsymbol{J} \times \boldsymbol{B}+\mu \nabla^{2} \boldsymbol{V} \\
\left.0=-\nabla \nabla \cdot \Pi^{\mathrm{neo}}\right\rangle_{\mathrm{srf}}, \\
\frac{3}{2} \frac{d_{\mathrm{i}} p_{\mathrm{i}}}{d t}-\frac{5}{2} p_{\mathrm{i}} \nabla \cdot \boldsymbol{V}_{\mathrm{i}}=-\nabla \cdot \boldsymbol{q}_{\mathrm{i}},
\end{gathered}
$$




$$
\frac{3}{2} \frac{d_{\mathrm{e}} p_{\mathrm{e}}}{d t}-\frac{5}{2} p_{\mathrm{e}} \nabla \cdot \boldsymbol{V}_{\mathrm{e}}=-\nabla \cdot \boldsymbol{q}_{\mathrm{e}},
$$

with $d / d t=\partial / \partial t+\boldsymbol{V}_{E} \cdot \nabla, \quad d_{\mathrm{i}} / d t=\partial / \partial t+\boldsymbol{V}_{\mathrm{i}} \cdot \nabla, \quad d_{\mathrm{e}} / d t$ $=\partial / \partial t+\boldsymbol{V}_{\mathrm{e}} \cdot \nabla$,

$$
\begin{gathered}
\boldsymbol{J}=\frac{c}{4 \pi} \nabla \times \boldsymbol{B} \\
\frac{1}{e n_{\mathrm{i}}} \boldsymbol{R}=\eta_{\|} \boldsymbol{J}_{\|}+\eta_{\perp} \boldsymbol{J}_{\perp}-\frac{\alpha_{T}}{e} \boldsymbol{b}(\boldsymbol{b} \cdot \nabla) T_{\mathrm{e}} \\
\frac{1}{n_{\mathrm{i}}} \boldsymbol{q}_{\mathrm{i}}=-\chi_{\| i} \boldsymbol{b} \cdot \nabla T_{\mathrm{i}}-\chi_{\perp \mathrm{i}}(\nabla-\boldsymbol{b}(\boldsymbol{b} \cdot \nabla)) T_{\mathrm{i}}+\frac{5}{2} \frac{c T_{\mathrm{i}}}{e B} \boldsymbol{b} \times \nabla T_{\mathrm{i}},
\end{gathered}
$$

$$
\begin{aligned}
\frac{1}{n_{\mathrm{e}}} \boldsymbol{q}_{\mathrm{e}}= & -\chi_{\| \mathrm{e}} \boldsymbol{b} \cdot \nabla T_{\mathrm{e}}-\chi_{\perp \mathrm{e}}(\nabla-\boldsymbol{b}(\boldsymbol{b} \cdot \nabla)) T_{\mathrm{e}} \\
& -\frac{\alpha_{T} T_{\mathrm{e}}}{e n_{\mathrm{e}}} \boldsymbol{J}_{\|}-\frac{5}{2} \frac{c T_{\mathrm{e}}}{e B} \boldsymbol{b} \times \nabla T_{\mathrm{e}},
\end{aligned}
$$

where $\boldsymbol{B}=\boldsymbol{B} \boldsymbol{b}$ is the magnetic field, $\boldsymbol{b}$ is the unit vector parallel to the magnetic field, $B=\boldsymbol{b} \cdot \boldsymbol{B}, \boldsymbol{E}$ is the electric field, $\boldsymbol{J}$ is the current density, $\boldsymbol{J}_{\|}=j_{\|} \boldsymbol{b}, j_{\|}=\boldsymbol{b} \cdot \boldsymbol{J}, \boldsymbol{J}_{\perp}=\boldsymbol{J}-\boldsymbol{J}_{\|}, \boldsymbol{V}=$ $\boldsymbol{V}_{\|}+\boldsymbol{V}_{E}$ is the single fluid velocity, $\boldsymbol{V}_{\|}=v_{\|} \boldsymbol{b}, v_{\|}=\boldsymbol{b} \cdot \boldsymbol{V}, \boldsymbol{V}_{E}$ is the $\mathrm{E} \times B$ drift velocity, $\boldsymbol{V}_{\mathrm{i}(\mathrm{e})}=\boldsymbol{V}+\boldsymbol{V}_{* \mathrm{i}(\mathrm{e})}, \boldsymbol{V}_{* \mathrm{i}(\mathrm{e})}$ is the ion (electron) diamagnetic drift velocity, $n_{\mathrm{i}(\mathrm{e})}$ is the ion (electron) density, $T_{\mathrm{i}(\mathrm{e})}$ is the ion (electron) temperature, $p_{\mathrm{i}(\mathrm{e})}$ is the ion (electron) pressure, $p=p_{\mathrm{i}}+p_{\mathrm{e}}, \Pi_{\mathrm{i}(\mathrm{e})}^{\text {neo }}$ is the anisotropic ion (electron) pressure tensor associated with the neoclassical viscosity, $\Pi^{\text {neo }}=\Pi_{\mathrm{i}}^{\text {neo }}+\Pi_{\mathrm{e}}^{\text {neo }}, \mu$ is the perpendicular anomalous viscosity coefficient, $\eta_{\|}$is the parallel resistivity, $\eta_{\perp}$ is the perpendicular resistivity, $\chi_{\| i \text { (e) }}$ is the parallel ion (electron) thermal diffusivity, $\chi_{\perp \mathrm{i}(\mathrm{e})}$ is the perpendicular ion (electron) thermal diffusivity, $\alpha_{\mathrm{T}}=0.71, m_{\mathrm{i}}$ is the ion mass, $e$ is the elementary charge, and $c$ is the velocity of the light. The bracket \langle\rangle$_{\text {srf }}$ denotes the average over the magnetic surface, which will be defined in the Subsection II B. In Eqs. (8)-(10), terms including a ratio of the gyro-frequency to the collision frequency and the Larmor radius are neglected for simplicity. To eliminate a term $m_{\mathrm{i}} n_{\mathrm{i}}\left(d_{\mathrm{i}} \boldsymbol{V}_{* \mathrm{i}} / d t\right)$ and the gyro-viscous term in Eq. (3), the so-called gyroviscous cancellation is taken into account. ${ }^{35,40}$ Residual terms due to imperfect cancellation are neglected for simplicity, although those appear in the model with hot ion fluids. ${ }^{38,39}$ In Eq. (4), the electron inertia is neglected for simplicity. According to the large amount of experimental observations in torus plasmas, both the perpendicular thermal diffusivity $\chi_{\perp \mathrm{i}(\mathrm{e})}$ and the viscosity coefficient $\mu$ are anomalously larger than those predicted by the classical and neoclassical theories, ${ }^{41,42}$ which might be due to microturbulence. The detailed modeling of the anomalous effects is still an open issue. ${ }^{43}$ Therefore, we treat these coefficients as phenomenological parameters. In addition, the neoclassical thermal transport is not considered, since that is typically smaller than the anomalous perpendicular thermal transport. Operating $(\boldsymbol{B} \cdot \nabla \times)$ to Eq. (3) eliminates the compressional Alfvén wave, and gives the Shear Alfvén law

$$
c \boldsymbol{B} \cdot(\nabla \times \boldsymbol{f}-2 \boldsymbol{\kappa} \times \boldsymbol{f})=B^{2} \boldsymbol{B} \cdot \nabla\left(\frac{J_{\|}}{B}\right)+2 c \boldsymbol{B} \times \boldsymbol{\kappa} \cdot \nabla p,
$$

where $\boldsymbol{f}=m_{\mathrm{i}} n_{\mathrm{i}}\left(d_{\mathrm{i}} \boldsymbol{V}_{E}+d \boldsymbol{V}_{\|} / d t\right)-\mu \nabla^{2} \boldsymbol{V}+\left\langle\nabla \cdot \Pi^{\text {neo }}\right\rangle_{\text {srf }}$ and $\boldsymbol{\kappa}=(\boldsymbol{b} \cdot \nabla) \boldsymbol{b}$ is the magnetic field line curvature.

\section{B. Stellarator magnetic field and ordering}

We consider a helical plasma with the averaged minor radius $a$ and the major radius $R_{0}$, using the toroidal coordinates $(R, \theta, \zeta)$, where $R$ is the major radial position, $\theta$ is the poloidal angle, and $\zeta$ is the toroidal angle. The magnetic field is given by

$$
\boldsymbol{B}=B_{0} \frac{R_{0}}{R} \hat{\zeta}+\boldsymbol{B}_{\mathrm{h}}+B_{\mathrm{dia}} \hat{\zeta}+\widetilde{\boldsymbol{B}}
$$

where $\left(B_{0} R_{0} / R\right) \hat{\zeta}$ is the toroidally axisymmetric magnetic field, $B_{0}$ is the toroidal magnetic field on the magnetic axis, $\hat{\zeta}$ represents the toroidal unit vector, $\boldsymbol{B}_{\mathrm{h}}$ is the toroidally nonaxisymmetric but helically symmetric part of the magnetic field, $B_{\text {dia }} \sim-B_{0} \beta / 2$ is the diamagnetic field, $\beta$ is the total plasma pressure normalized by the toroidal magnetic pressure $B_{0}^{2} / 8 \pi$, and $\widetilde{\boldsymbol{B}}$ is the magnetic field perturbation which involves the externally applied RMP. The magnetic field perturbation is given by

$$
\widetilde{\boldsymbol{B}}=\nabla \times \boldsymbol{A},
$$

where $\boldsymbol{A}=A \hat{\zeta}$ is the vector potential perturbation. In the following, the cylindrical coordinates $(r, \theta, z)$ are used for convenience, where $r$ is defined by $R=R_{0}+r \cos \theta, z=R_{0} \zeta$ and unit vectors are $\{\hat{\boldsymbol{r}}, \hat{\theta}, \hat{z}\}$. Introducing $\boldsymbol{B}=\left(b_{r}, b_{\theta}, B_{0}+b_{z}\right)$, the magnetic field line is determined by

$$
\frac{d r_{\mathrm{m}}}{b_{r}}=\frac{r d \theta_{\mathrm{m}}}{b_{\theta}}=\frac{d z_{\mathrm{m}}}{B_{0}+b_{z}}=\frac{d l_{\mathrm{m}}}{B},
$$

where $\left(r_{\mathrm{m}}, \theta_{\mathrm{m}}, z_{\mathrm{m}}\right)$ is the position along the magnetic field line and $l_{\mathrm{m}}$ is the distance along the magnetic field line. The magnetic surface average is defined by

$$
\langle f\rangle_{\mathrm{srf}}=\frac{\oint \frac{f}{B} d l_{\mathrm{m}}}{\oint \frac{1}{B} d l_{\mathrm{m}}},
$$

where $f$ is arbitrary. We assume the so-called stellarator expansion ordering

$$
\epsilon_{\mathrm{t}} \sim \epsilon_{\mathrm{h}}^{2} \sim \beta,
$$

where $\epsilon_{\mathrm{t}}=r / R_{0}, \epsilon_{\mathrm{h}}=\left|\boldsymbol{B}_{\mathrm{h}}\right| / B_{0}$, and $\left|\boldsymbol{B}_{\mathrm{h}}\right|$ indicates the amplitude of the ripple magnetic field. In order $\epsilon_{\mathrm{h}}$, the magnetic field is expressed as $\boldsymbol{B}_{\mathrm{h}}=\nabla \Phi$, where $\Phi$ is a scalar variable, since the magnetic field in the current-less limit is curl-less. The Gauss's law for magnetism $\nabla^{2} \Phi=0$ gives $\Phi=\sum_{j, k}$ $\Phi_{j, k} I_{k}\left(j r / R_{0}\right) \exp \left\{i\left(k \theta+j z / R_{0}\right)\right\}$, where $I_{k}$ is the modified Bessel function. Here, a dominant component

$$
\Phi=\Phi_{l, M} I_{l}\left(\frac{M r}{R_{0}}\right) \sin \left(l \theta+\frac{M z}{R_{0}}\right)
$$

is considered, where $\Phi_{l, M}$ is a coefficient and $\{l, M\}$ are the pole and pitch numbers of the helically winding coil, 
respectively. The so-called drift ordering, where the plasma velocity is comparable to the diamagnetic drift velocity, is assumed to justify a fluid model with the transport closure: ${ }^{35}$ $v_{\|} \sim \boldsymbol{V}_{E} \sim \boldsymbol{V}_{* \mathrm{i}} \sim \boldsymbol{V}_{* \mathrm{e}} \sim O\left(\epsilon_{\mathrm{t}}\right)$. The assumption of the large aspect ratio leads to $\partial_{r} \sim \partial_{\theta} \sim O\left(\epsilon_{\mathrm{t}}^{0}\right)$ and $\partial_{z} \sim O\left(\epsilon_{\mathrm{t}}\right)$. The low- $\beta$ assumption implies $p \sim p_{\mathrm{i}} \sim p_{\mathrm{e}} \sim n_{\mathrm{i}} \sim n_{\mathrm{e}} \sim T_{\mathrm{i}} \sim T_{\mathrm{e}}$ $\sim O(\beta)$. We only focus on the slow time variation comparable to the drift frequency: $\partial_{t} \sim \boldsymbol{V}_{* \mathrm{i}} \cdot \nabla \sim \boldsymbol{V}_{* \mathrm{e}} \cdot \nabla \sim O\left(\epsilon_{\mathrm{t}}\right)$. The electro-magnetic fields are ordered as $\boldsymbol{E} \sim \boldsymbol{J} \sim A$ $\sim O\left(\epsilon_{\mathrm{t}}\right)$. The transport coefficients are ordered as $\mu \sim \eta_{\|} \sim$ $\eta_{\perp} \sim \chi_{\perp \mathrm{i}} \sim \chi_{\perp \mathrm{e}} \sim O\left(\epsilon_{\mathrm{t}}\right)$ and $\chi_{\| \mathrm{i}} \sim \chi_{\| \mathrm{e}} \sim O\left(\epsilon_{\mathrm{t}}^{-1}\right)$. To include the neoclassical viscosity, we assume $\Pi_{\mathrm{i}}^{\text {neo }} \sim \Pi_{\mathrm{e}}^{\text {neo }} \sim \Pi^{\text {neo }}$ $\sim O\left(\epsilon_{\mathrm{t}}^{2}\right)$.

\section{Reduced fluid model}

Considering Eq. (17), the $z_{\mathrm{m}}$ dependence is separated into the slowly changing variable $z$ and the rapidly changing variable $Z=M z / R_{0}$. Therefore, the $z_{\mathrm{m}}$ derivative is separated as $\partial_{z}+\partial_{Z}$, where $\partial_{z} \sim O\left(\epsilon_{\mathrm{h}}^{2}\right)$ and $\partial_{\mathrm{Z}} \sim O\left(\epsilon_{\mathrm{h}}^{0}\right)$. The toroidal average is defined by ${ }^{44,45}$

$$
\bar{f}=\frac{1}{2 \pi} \int_{0}^{2 \pi} f d Z,
$$

where $f$ is arbitrary. Similarly, the radial and poloidal positions along the magnetic field are split into the average and fluctuating parts

$$
\begin{gathered}
r_{\mathrm{m}}=r(\theta, z)+\delta r(r, \theta, z, Z), \\
\theta_{\mathrm{m}}=\theta(r, z)+\delta \theta(r, \theta, z, Z),
\end{gathered}
$$

where $\delta r / r \sim \delta \theta / \theta \sim O\left(\epsilon_{\mathrm{h}}\right)$. Henceforth, the coordinates $(r, \theta, z)$ represent the position on the average magnetic field. A reduced fluid model is given by the toroidal averaging of Eqs. (1)-(6) and (11) with the ordering shown in the Subsection II B. In the averaging procedure, the average magnetic field and the average magnetic field curvature are given after the lengthy calculation. ${ }^{44,45}$ In particular, the careful treatment of the curvature term in Eq. (11) is necessary, since the nonlinear coupling of the curvature and the magnetic field is explicitly included. We neglect the nonlinear coupling of $\boldsymbol{B}_{\mathrm{h}}$ in the parallel thermal transport terms in Eqs. (5) and (6) for simplicity. Other parts of the derivation follow those in the reduced fluid model in tokamaks. ${ }^{35}$ The reduced fluid model is given by

$$
\begin{gathered}
\frac{c^{2}}{4 \pi v_{\mathrm{A}}^{2}} \frac{D_{\mathrm{i}}}{D t} \nabla_{\perp}^{2} \phi=\nabla_{\|} j_{\|}+\frac{2 c}{B_{0}} \hat{z} \cdot \overline{\boldsymbol{\kappa}} \times \nabla_{\perp} p+\frac{c^{2} \mu}{4 \pi v_{\mathrm{A}}^{2}} \nabla_{\perp}^{4} \phi \\
+\frac{c}{B_{0}} \hat{z} \cdot \nabla_{\perp} \times{\overline{\left\langle\nabla \cdot \Pi^{\mathrm{neo}}\right\rangle_{\mathrm{srf}}}} \\
\frac{1}{c} \frac{\partial A}{\partial t}=-\widetilde{\nabla_{\|} \phi}-\frac{1}{e n_{0}} \widetilde{\nabla_{\|} p_{\mathrm{e}}}-\frac{\alpha_{T}}{e} \widetilde{\nabla_{\|} T_{\mathrm{e}}}-\eta_{\|}{\widetilde{j_{\|}}}_{\|} \\
-\frac{1}{e n_{0}} \hat{z} \cdot \widetilde{\left\langle\nabla \cdot \Pi_{\mathrm{e}}^{\mathrm{neo}}\right\rangle_{\mathrm{sr}}}, \\
\frac{D}{D t} v_{\|}=-\frac{1}{m_{i} n_{0}} \nabla_{\|} p+\mu \nabla_{\perp}^{2} v_{\|},
\end{gathered}
$$

$$
\begin{gathered}
\frac{D}{D t} n_{\mathrm{e}}=\frac{1}{e} \nabla_{\| j_{\|}}+n_{0} \nabla_{\|} v_{\|}, \\
\frac{3}{2} \frac{D}{D t} p_{\mathrm{e}}=\frac{\alpha_{T}^{\prime} T_{\mathrm{e} 0}}{e} \nabla_{\| j_{\|}}+\frac{5}{2} n_{0} T_{\mathrm{e} 0} \nabla_{\|} v_{\|}+\chi_{\| \mathrm{e}} \nabla_{\|}^{2} p_{\mathrm{e}}+\chi_{\perp \mathrm{e}} \nabla_{\perp}^{2} p_{\mathrm{e}} \\
\frac{3}{2} \frac{D}{D t} p_{i}=\frac{5}{2} n_{0} T_{\mathrm{i} 0} \nabla_{\|} v_{\|}+\chi_{\| \mathrm{i}} \nabla_{\|}^{2} p_{\mathrm{i}}+\chi_{\perp \mathrm{i}} \nabla_{\perp}^{2} p_{\mathrm{i}}
\end{gathered}
$$

where $j_{\|}=j_{\| 0}+\widetilde{j_{\|}}, \widetilde{j}_{\|}=-(c / 4 \pi) \nabla_{\perp}^{2} A, \phi$ is the electrostatic potential, $v_{A}$ is the Alfvén velocity, and $\alpha_{T}^{\prime}=5 / 2+\alpha_{T}$. The suffix " 0 " indicates the unperturbed equilibrium value, and the tilde represents the perturbation with the same mode number as $A$. We assumed the quasi-neutral condition between the ion and electron densities, $n_{\mathrm{i}}=n_{\mathrm{e}}$, and defined $n_{\mathrm{i} 0}=n_{\mathrm{e} 0}=n_{0}$. The derivatives are defined by

$$
\begin{gathered}
\frac{D}{D t}=\frac{\partial}{\partial t}+[\phi,], \\
\frac{D_{\mathrm{i}}}{D t}=\frac{\partial}{\partial t}+\left[\phi-\frac{1}{e n_{0}} p_{\mathrm{i}},\right], \\
\nabla_{\|}=\frac{\partial}{\partial z}+\frac{l}{R_{0}} \frac{\partial}{\partial \theta}-\frac{1}{B_{0}}[A,], \\
\nabla_{\perp}=\hat{\boldsymbol{r}} \frac{\partial}{\partial r}+\hat{\theta} \frac{1}{r} \frac{\partial}{\partial \theta},
\end{gathered}
$$

where $[f, g]=\hat{z} \cdot \nabla_{\perp} f \times \nabla_{\perp} g$ for arbitrary $f$ and $g$. The average rotational transform normalized by $2 \pi$, which is the inverse of the safety factor $q ; \imath=1 / q$, is given by ${ }^{44,45}$

$$
\imath=\frac{R_{0} \bar{B}_{\theta}}{r B_{0}},
$$

where $\quad \bar{B}_{\theta}=\hat{\theta} \cdot \nabla A_{0} \times \hat{z}, \quad A_{0}=\left(1 / 2 B_{0}\right) \hat{z} \cdot \overline{\nabla \Phi \times \nabla\langle\Phi\rangle_{\mathrm{ta}}}$,

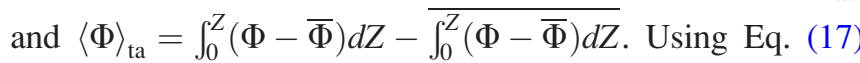
gives

$$
l(r)=\frac{l \Phi_{l M}^{2} R_{0}^{2}}{4 M B_{0}^{2}} \frac{1}{r} \frac{d}{d r}\left[\frac{1}{r} \frac{d}{d r} I_{l}^{2}(x)\right] .
$$

where $x=M r / R_{0}$. Since the pressure-gradient term in $\boldsymbol{\kappa}=$ $\nabla B / B+\left(4 \pi / B^{2}\right) \nabla p$ vanishes in Eq. (11), an average of $\nabla B / B$ is only required. Then, the average curvature is given by $^{44,45}$

$$
\overline{\boldsymbol{\kappa}}=\nabla_{\perp}\left(-\frac{r}{R_{0}} \cos \theta+\frac{1}{2 B_{0}^{2}} \overline{|\nabla \Phi|^{2}}\right) .
$$

Some manipulations of Eq. (32) with the modified Bessel equation give the following relation

$$
\frac{1}{B_{0}^{2}} \overline{|\nabla \Phi|^{2}}=1+\frac{M}{R_{0}^{2} l}\left(r^{2} \iota+2 \int_{0}^{r} r l d r\right) .
$$

Finally, using Eq. (34) rewrites the average curvature as

$$
\overline{\boldsymbol{\kappa}}=\nabla_{\perp}\left(-\frac{r}{R_{0}} \cos \theta\right)+\kappa_{\mathrm{h}} \hat{\boldsymbol{r}},
$$


with

$$
\kappa_{\mathrm{h}}=\frac{M}{2 l R_{0}^{2}} r l(4-s)
$$

where $s=-(r / \imath)(d \imath / d r)$ is the magnetic shear.

\section{Neoclassical closure}

The neoclassical particle transport in stellarators is mainly due to the interaction between the passing particle and the banana particles trapped by the ripple toroidal magnetic field. Note that toroidal magnetic fields by magnetic islands are smaller than that of the ambient helical field $\boldsymbol{B}_{\mathrm{h}}$. Thus, in strongly non-axisymmetric stellarators, the neoclassical particle flux might be well approximated by that in the absence of magnetic islands. Radial neoclassical particle fluxes of ions and electrons averaged over the original magnetic surface, $\Gamma_{\mathrm{i}}$ and $\Gamma_{\mathrm{e}}$, are calculated by the drift-kinetic equation with the drift ordering and the transport ordering, ${ }^{35}$ by integrating the radial velocity weighted by distribution function perturbation in the whole velocity space. A generalized expression of the particle flux is obtained by connecting the results in many collisionality regime, depending on the $E \times B$ drift frequency, the $\nabla B$ drift frequency, and the collision frequency. In this study, we employ the model in Ref. 46 in the absence of the $\nabla B$ drift. The neglect of the $\nabla B$ drift implies that the strong $E \times B$ drift prevents the super-banana orbit, and such parameter regime is of interest. Further, we assume that $B \approx B_{0}$ and the collision frequency is much slower than the thermal velocity transit time. Then, we obtain the neoclassical radial particle flux

$$
\begin{aligned}
\Gamma_{\alpha}^{\text {neo }}= & \epsilon_{\mathrm{t}}^{2} \epsilon_{\mathrm{h}}^{3 / 2} V_{\perp \alpha}^{2} n_{\alpha} \int_{0}^{\infty} d x \frac{\nu_{\alpha} x^{5 / 2} \exp (-x)}{c_{1} \epsilon_{\mathrm{t}} \epsilon_{\mathrm{h}} \omega_{E}^{2}+c_{2} \nu_{\alpha}^{2}} \\
& \times\left[\frac{n_{\alpha}^{\prime}}{n_{\alpha}}-\frac{e_{\alpha} E_{r}}{T_{\alpha}}+\left(x-\frac{3}{2}\right) \frac{T_{\alpha}^{\prime}}{T_{\alpha}}\right]
\end{aligned}
$$

for $\alpha(=\mathrm{i}, \mathrm{e})$ species, where $x$ is the square of the velocity normalized by the thermal velocity, $E_{r}$ is the radial electric field, the prime indicates the radial derivative, $V_{\perp \alpha}=c T_{\alpha} / e_{\alpha} B_{0} r, e_{\mathrm{i}(\mathrm{e})}=e(-e), \omega_{E}=-c E_{r} / r B_{0}, \nu_{\alpha}$ is the collision frequency, $c_{1}=1.67$ (Ref. 46), and $c_{2}=3$. $^{46}$ Although the collision frequencies are functions of $x$, in general, we replace them to those measured at the thermal velocities. Using $\int_{0}^{\infty} d x x^{5 / 2} e^{-x}=15 \sqrt{\pi} / 8$ and $\int_{0}^{\infty} d x x^{7 / 2} e^{-x}$ $=105 \sqrt{\pi} / 16$, we finally obtain

$$
\Gamma_{\alpha}^{\text {neo }}=\frac{c_{3} \epsilon_{\mathrm{t}}^{2} \epsilon_{\mathrm{h}}^{3 / 2} V_{\perp \alpha}^{2} n_{\alpha} \nu_{\alpha}}{c_{1} \epsilon_{\mathrm{t}} \epsilon_{\mathrm{h}} \omega_{E}^{2}+c_{2} \nu_{\alpha}^{2}}\left(\frac{n_{\alpha}^{\prime}}{n_{\alpha}}-\frac{e_{\alpha} E_{r}}{T_{\alpha}}+\frac{2 T_{\alpha}^{\prime}}{T_{\alpha}}\right) .
$$

where $c_{3}=15 \sqrt{\pi} / 8$. Operating $(\hat{\boldsymbol{z}} \times)$ to Eq. (3) and applying the toroidal averaging, we model the neoclassical perpendicular current

$$
\overline{\boldsymbol{j}_{\perp}^{\mathrm{neo}}}=\frac{c}{B_{0}} \hat{\boldsymbol{z}} \times{\overline{\left\langle\nabla \cdot \Pi^{\mathrm{neo}}\right\rangle}}_{\mathrm{srf}}=e\left(\Gamma_{\mathrm{i}}^{\mathrm{neo}}-\Gamma_{\mathrm{e}}^{\mathrm{neo}}\right) \hat{\boldsymbol{r}} .
$$

Operating $\left(\nabla_{\perp} \cdot\right)$ to Eq. (39) gives

$$
\frac{c}{B_{0}} \hat{z} \cdot \nabla_{\perp} \times{\overline{\left\langle\nabla \cdot \Pi^{\text {neo }}\right\rangle}}_{\text {srf }}=\frac{e}{r} \frac{\partial}{\partial r}\left[r\left(\Gamma_{\mathrm{i}}^{\text {neo }}-\Gamma_{\mathrm{e}}^{\text {neo }}\right)\right],
$$

which is the neoclassical closure in Eq. (21).

For consistency with the neoclassical theory, the parallel force balance $\left\langle\boldsymbol{B} \cdot \nabla \cdot \Pi^{\text {neo }}\right\rangle_{\text {srf }}=0$ should be taken into account. This is why the neoclassical viscosity does not appear in Eq. (23). In Eq. (23), only the lowest order of this balance with the toroidal averaging

$$
\hat{z} \cdot{\overline{\left\langle\nabla \cdot \Pi^{\text {neo }}\right\rangle}}_{\text {srf }}=0
$$

is important, since the neoclassical viscosity is of order $O\left(\epsilon_{\mathrm{t}}^{2}\right)$ in our ordering and the correction of order $O\left(\epsilon_{\mathrm{t}}^{3}\right)$ is lost.

In this study, we neglect the influence of the perturbed bootstrap current, and simply assume

$$
-\frac{1}{e n_{0}} \hat{z} \cdot{\widetilde{\left\langle\nabla \cdot \Pi_{\mathrm{e}}^{\text {neo }}\right\rangle_{\mathrm{srf}}}}_{\left\langle{ }^{\prime}\right.}=0
$$

in the lowest order, and the equilibrium bootstrap current is not included in $j_{\| 0}$. The neglect of the perturbed bootstrap current is mainly due to a fact that the stellarator equilibrium is based on the toroidal-current-less condition in most cases. The influence of the perturbed bootstrap current will be briefly discussed in Sec. III F.

\section{DERIVATION OF MODEL EQUATIONS}

\section{A. Asymptotic matching}

We assume that the perturbation is dominated by a single mode with the poloidal mode number $m$ and the toroidal mode number $n$, which is resonant at the rational surface $l_{\mathrm{s}}=\imath\left(r_{\mathrm{s}}\right)=n / m$, where $r_{\mathrm{s}}$ is the average minor radial position of the rational surface. There exists a boundary layer near the rational surface, where non-ideal MHD effects become important and the magnetic reconnection is driven by the resonant mode. We introduce the inner-layer current perturbation $\widetilde{j}_{\| \text {in }}=-(c / 4 \pi) \nabla_{\perp}^{2} A_{\text {in }}$ and the outer layer current perturbation $\widetilde{j}_{\| \text {out }}=-(c / 4 \pi) \nabla_{\perp}^{2} A_{\text {out }}$. Hereafter, the subscripts in' and 'out' denote the inner-layer quantity and the outer-layer quantity, respectively. Here, $A_{\text {in }}$ is written as

$$
\begin{gathered}
A_{\text {in }}=\psi_{\mathrm{s}} \cos \Theta, \\
\Theta=m \theta-\frac{n z}{R_{0}}+\Delta \Theta(t),
\end{gathered}
$$

where $\psi_{\mathrm{s}}$ is the amplitude at the rational surface, $\Theta$ is the phase angle, and $\Delta \Theta$ is the time dependent part of the phase angle. We consider integrals of the current perturbation multiplied by $\cos \Theta$ and $\sin \Theta$ across the boundary layer. The inner-layer integral is $\int_{-\infty}^{\infty} d x \int_{-\pi}^{\pi} d \Theta$ and the outer-layer integral is $\int_{-w / 2}^{+w / 2} d x \int_{-\pi}^{\pi} d \Theta$, where $x=r-r_{\mathrm{s}}, w=4 \sqrt{L_{s} \psi_{\mathrm{s}} / B_{0}}$ is the magnetic island width, $L_{s}=\sigma_{s} R_{0} / l_{\mathrm{s}} s_{\mathrm{s}}, \sigma_{s}=\operatorname{sgn}\left(s_{\mathrm{s}}\right)$, and $s_{\mathrm{S}}=s\left(r_{\mathrm{s}}\right)$. Near the rational surface, it is reasonable to approximate $\nabla_{\perp}^{2} A_{\text {out }} \rightarrow \partial_{x}^{2} A_{\text {out }}$. Then, so-called the cosine and sine matchings are written as ${ }^{7-9}$ 


$$
\begin{aligned}
& \int_{-\infty}^{\infty} d x \int_{-\pi}^{\pi} d \Theta \widetilde{j}_{\| \text {in }} \cos \Theta=-\frac{c}{4} \psi_{\mathrm{s}} \Delta_{\mathrm{c}}^{\prime}, \\
& \int_{-\infty}^{\infty} d x \int_{-\pi}^{\pi} d \Theta \widetilde{j}_{\| \text {in }} \sin \Theta=-\frac{c}{4} \psi_{\mathrm{s}} \Delta_{\mathrm{s}}^{\prime},
\end{aligned}
$$

with

$$
\begin{aligned}
& \Delta_{\mathrm{c}}^{\prime}=\frac{1}{\pi \psi_{\mathrm{s}}} \int_{-\pi}^{\pi} d \Theta\left[\frac{\partial A_{\text {out }}}{\partial x}\right]_{-w / 2}^{w / 2} \cos \Theta \\
& \Delta_{\mathrm{s}}^{\prime}=\frac{1}{\pi \psi_{\mathrm{s}}} \int_{-\pi}^{\pi} d \Theta\left[\frac{\partial A_{\text {out }}}{\partial x}\right]_{-w / 2}^{w / 2} \sin \Theta
\end{aligned}
$$

where the prime indicates the radial derivative.

For analytical traceability, we assume that $\psi_{s}$ is constant in the inner layer, i.e., the so-called constant- $\psi$ approximation. Strictly speaking, this approximation might be reasonable when $\left|w \Delta_{\mathrm{c}}^{\prime}\right|,\left|w \Delta_{\mathrm{s}}^{\prime}\right| \ll 1$ and the resistive diffusion inside islands is much faster than the rotation frequency of islands. Although we use the approximation throughout this study, the extension of the theory to that in the non-constant- $\psi$ regime is left as a future work.

\section{B. Outer-layer calculation}

Far from the rational surface, i.e., in the outer-layer, $A_{\text {out }}$ is given by the perturbed MHD equilibrium $\boldsymbol{J} \times \boldsymbol{B}=c \nabla p$ and Eq. (7). Operating $(\boldsymbol{B} \cdot \nabla \times)$ and $(\boldsymbol{B} \cdot)$ to $\boldsymbol{J} \times \boldsymbol{B}=c \nabla p$, and applying the toroidal averaging give Eqs. (21) and (23) in the ideal MHD limit

$$
\begin{gathered}
\nabla_{\| j_{\| \text {out }}+}+\frac{2 c}{B_{0}} \hat{z} \cdot \overline{\boldsymbol{\kappa}} \times \nabla_{\perp} p_{\text {out }}=0, \\
\nabla_{\|} p_{\text {out }}=0
\end{gathered}
$$

where $j_{\| \text {out }}=j_{\| 0}+\widetilde{j}_{\| \text {out }}$. Substituting $p_{\text {out }}$ in the linearized Eq. (50) into the linearized Eq. (49) gives

$$
\frac{1}{r} \frac{\partial}{\partial r}\left(r \frac{\partial A_{\text {out }}}{\partial r}\right)-\left(k_{\theta}^{2}+\frac{4 \pi k_{\theta} j_{\| 0}^{\prime}}{c B_{0} k_{\|}}+\frac{4 \pi \kappa_{\mathrm{h}} k_{\theta}^{2} p_{0}^{\prime}}{B_{0}^{2} k_{\|}^{2}}\right) A_{\text {out }}=0,
$$

where $p_{0}$ is the unperturbed pressure, $k_{\theta}=m / r$, and $k_{\|}=(m \imath-n) / R_{0}$. The last term on the left-hand side (LHS) of Eq. (51) gives rise to the perturbed Pfirsch-Schlüter current in stellarators. In the presence of magnetic islands and RMP, $A_{\text {out }}$ satisfies the boundary condition: $A_{\text {out }}(0)=0$, $A_{\text {out }}\left(r_{\mathrm{s}} \pm w / 2\right)=\psi_{\mathrm{s}} \cos \Theta$, and $A_{\text {out }}(a)=\psi_{a} \cos (\Theta-\Delta \Theta)$. According to Ref. 7, without any loss of generality, $A_{\text {out }}$ is separated as

$$
A_{\text {out }}=\psi_{\mathrm{m}}(r) \cos \Theta+\psi_{\mathrm{c}}(r) \cos (\Theta-\Delta \Theta) .
$$

Equation (51) is rewritten as

$$
\frac{1}{r} \frac{\partial}{\partial r}\left(r \frac{\partial \psi_{\mathrm{m}(\mathrm{c})}}{\partial r}\right)-\left(k_{\theta}^{2}+\frac{4 \pi k_{\theta} j_{\| 0}^{\prime}}{c B_{0} k_{\|}}+\frac{4 \pi \kappa_{\mathrm{h}} k_{\theta}^{2} p_{0}^{\prime}}{B_{0}^{2} k_{\|}^{2}}\right) \psi_{\mathrm{m}(\mathrm{c})}=0
$$

and the boundary condition becomes $\psi_{\mathrm{m}}(0)=0, \quad \psi_{\mathrm{m}}$ $\left(r_{\mathrm{s}} \pm w / 2\right)=\psi_{\mathrm{s}}, \psi_{\mathrm{m}}(a)=0, \psi_{\mathrm{c}}(0)=0, \psi_{\mathrm{c}}\left(r_{\mathrm{s}} \pm w / 2\right)=0$, and $\psi_{\mathrm{c}}(a)=\psi_{a}$. Using $\psi_{\mathrm{m}}$ and $\psi_{\mathrm{c}}$ yields

$$
\begin{gathered}
\Delta_{\mathrm{c}}^{\prime}=\Delta_{\text {mode }}^{\prime}+\Delta_{\text {coil }}^{\prime} \cos \Delta \Theta, \\
\Delta_{\mathrm{s}}^{\prime}=\Delta_{\text {coil }}^{\prime} \sin \Delta \Theta,
\end{gathered}
$$

with

$$
\begin{aligned}
\Delta_{\text {mode }}^{\prime} & =\frac{1}{\psi_{\mathrm{s}}}\left[\frac{\partial \psi_{\mathrm{m}}}{\partial x}\right]_{-w / 2}^{w / 2} \\
\Delta_{\text {coil }}^{\prime} & =\frac{1}{\psi_{\mathrm{s}}}\left[\frac{\partial \psi_{\mathrm{c}}}{\partial x}\right]_{-w / 2}^{w / 2}
\end{aligned}
$$

In the limit of $j_{\| 0}=0$ and $\kappa_{\mathrm{h}}=0$, general solutions of Eq. (53) are $\psi_{\mathrm{m}}=C_{1} r^{m}+C_{2} r^{-m}$ and $\psi_{\mathrm{c}}=D_{1} r^{m}+D_{2} r^{-m}$, where $C_{1}, C_{2}, D_{1}$, and $D_{2}$ in $0 \leq r \leq r_{\mathrm{s}}$ and those $r_{\mathrm{s}} \leq r \leq a$ are determined by the boundary condition. Then, we obtain $\Delta_{\text {mode }}^{\prime}$ and $\Delta_{\text {coil }}^{\prime}$ in the limit of $j_{\| 0}=0$ and $\kappa_{\mathrm{h}}=0$

$$
\begin{gathered}
\Delta_{\text {mode }, 0}^{\prime}=-\frac{2 k_{\theta \mathrm{s}}}{1-r_{s}^{2 m} / a^{2 m}}=\Delta_{0}^{\prime}, \\
\Delta_{\text {coil }, 0}^{\prime}=-\Delta_{0}^{\prime} \frac{w_{\mathrm{v}}^{2}}{w^{2}},
\end{gathered}
$$

where $k_{\theta \mathrm{s}}=m / r_{\mathrm{s}}, \Delta_{0}^{\prime}$ is the tearing mode stability parameter in the currentless and straight stallarator, and the correction of order $w / r_{\mathrm{s}}$ is neglected. The vacuum island solution $w=w_{\mathrm{v}}$, where the vacuum island width is defined by $w_{\mathrm{v}}=4 \sqrt{\left(r_{\mathrm{s}} / a\right)^{m}\left(L_{s} \psi_{a} / B_{0}\right)}, \quad$ is given by $\Delta_{\text {mode }, 0}^{\prime}+$ $\Delta_{\text {coil }, 0}^{\prime} \cos \Delta \Theta=0$ with $\Delta \Theta=0$. In the small $j_{\| 0}$ and $\kappa_{\mathrm{h}}$ limit, we can expand $\psi_{\mathrm{m}}=\psi_{\mathrm{m}, 0}+\delta \psi_{\mathrm{m}}, \psi_{\mathrm{c}}=\psi_{\mathrm{c}, 0}+\delta \psi_{\mathrm{c}}$, $\Delta_{\text {mode }}^{\prime}=\Delta_{\text {mode }, 0}^{\prime}+\delta \Delta_{\text {mode }}^{\prime}$, and $\Delta_{\text {coil }}^{\prime}=\Delta_{\text {coil }, 0}^{\prime}+\delta \Delta_{\text {coil }}^{\prime}$. The lowest order of Eq. (53) near the rational surface gives $\psi_{\mathrm{m}, 0}=\psi_{\mathrm{s}}$ and $\psi_{\mathrm{c}, 0}=0$. In the first order, Eq. (53) near the rational surface is

$$
\frac{\partial^{2}}{\partial x^{2}} \delta \psi_{\mathrm{m}(\mathrm{c})}-\left(\frac{4 \pi k_{\theta \mathrm{s}} j_{\| 0 \mathrm{~s}}^{\prime}}{c B_{0} k_{\| \mathrm{s}}^{\prime} x}+\frac{4 \pi \kappa_{\mathrm{hs}} k_{\theta \mathrm{s}}^{2} p_{0 \mathrm{~s}}^{\prime}}{B_{0}^{2} k_{\| \mathrm{s}}^{\prime 2} x^{2}}\right) \psi_{\mathrm{m}(\mathrm{c}), 0}=0,
$$

where $j_{\| 0 \mathrm{~s}}^{\prime}=j_{\| 0}^{\prime}\left(r_{\mathrm{s}}\right), \kappa_{\mathrm{hs}}=\kappa_{\mathrm{h}}\left(r_{\mathrm{s}}\right), p_{0 \mathrm{~s}}^{\prime}=p_{0}^{\prime}\left(r_{\mathrm{s}}\right), k_{\| \mathrm{s}}^{\prime}=k_{\|}^{\prime}\left(r_{\mathrm{s}}\right)$, and $k_{\|} \approx k_{\| s}^{\prime} x$ is used. Operating $\int_{-w / 2}^{w / 2} d x$ to Eq. (60) yields

$$
\begin{aligned}
\delta \Delta_{\text {mode }}^{\prime} & =\frac{1}{\psi_{\mathrm{s}}}\left[\frac{\partial}{\partial x} \delta \psi_{\mathrm{m}}\right]_{-w / 2}^{w / 2}=\frac{2 D}{w} \\
\delta \Delta_{\text {coil }}^{\prime} & =\frac{1}{\psi_{\mathrm{s}}}\left[\frac{\partial}{\partial x} \delta \psi_{\mathrm{c}}\right]_{-w / 2}^{w / 2}=0
\end{aligned}
$$

with

$$
D=-\frac{8 \pi \kappa_{\mathrm{hs}} L_{s}^{2} p_{0 \mathrm{~s}}^{\prime}}{B_{0}^{2}}
$$

where $k_{\|_{\mathrm{s}}}^{\prime}=-\sigma_{s} k_{\theta \mathrm{s}} / L_{s}$ is used. Note that $D$ is an approximate value of the conventional resistive interchange mode parameter $D_{\mathrm{R}}$ or $E+F^{4,30-32}$ in the sense of the toroidal averaging. 


\section{Inner layer calculation}

The parallel derivative Eq. (29) near the rational surface is given by

$$
\nabla_{\| \text {in }}=-\left.\sigma_{s} \frac{k_{\theta s}}{L_{s}} x \frac{\partial}{\partial \Theta}\right|_{x}-\left.\frac{k_{\theta s} \psi_{s}}{B_{0}} \sin \Theta \frac{\partial}{\partial x}\right|_{\Theta}=-\left.\sigma_{s} \frac{k_{\theta s}}{L_{s}} x \frac{\partial}{\partial \Theta}\right|_{\Omega},
$$

where $\Omega$ is the helical flux function normalized by $\sigma_{s} \psi_{\mathrm{s}}$

$$
\Omega=\frac{8 x^{2}}{w^{2}}+\sigma_{s} \cos \Theta .
$$

The O-point and separatrix of magnetic correspond to $\Omega=-1$ and $\Omega=1$, respectively. Using the Jacobian $J=|\partial(x, \Theta)| /|\partial(\Omega, \Theta)|=\sigma_{s} w^{2} / 16 x$, the cosine and sine integrals in the $(x, \Theta)$-coordinates are transformed into those in the $(\Omega, \Theta)$-coordinates as

$$
\begin{aligned}
& \int_{-\infty}^{\infty} d x \int_{-\pi}^{\pi} d \Theta f \cos \Theta=\frac{\pi w}{\sqrt{2}} \int_{-1}^{\infty} d \Omega\langle f \cos \Theta\rangle_{\Omega}, \\
& \int_{-\infty}^{\infty} d x \int_{-\pi}^{\pi} d \Theta g \sin \Theta=\frac{\sigma_{s} L_{s}}{k_{\theta \mathrm{s}}} \int_{-1}^{\infty} d \Omega\left(\oint_{\Omega, x>0}+\oint_{\Omega, x<0}\right) \\
& \times \nabla_{\| \text {in }} g d \Theta \text {, }
\end{aligned}
$$

where $f$ and $g$ are arbitrary, and $\left.(\partial x / \partial \Theta)\right|_{\Omega}=\left(w^{2} / 16 x\right)$ $\sin \Theta$ and Eq. (64) are used in Eq. (67). The contour integral along the constant- $\Omega$ in Eq. (66) is defined by

$$
\langle h\rangle_{\Omega}=\frac{1}{4 \pi}\left(\oint_{\Omega(x>0)}+\oint_{\Omega(x<0)}\right) \frac{\sigma_{x} h}{\sqrt{\Omega-\sigma_{s} \cos \Theta}} d \Theta,
$$

where $\sigma_{x}=\operatorname{sgn}(x)$ and $h$ is arbitrary. It is confirmed $\left\langle\nabla_{\| \text {in } f}\right\rangle_{\Omega}=0$ for arbitrary $f$. Then, the cosine and sine matching Eqs. (45) and (46) are rewritten as

$$
\begin{aligned}
& \int_{-1}^{\infty} d \Omega\left\langle\widetilde{j}_{\| \text {in }} \cos \Theta\right\rangle_{\Omega}=-\frac{c \psi_{s}}{2 \sqrt{2} \pi w} \Delta_{\mathrm{c}}^{\prime}, \\
& \int_{-1}^{\infty} d \Omega \oint_{\Omega} d \Theta \nabla_{\| \text {in }} \widetilde{j}_{\| \text {in }}=-\frac{\sigma_{s} c k_{\theta s} \psi_{s}}{4 L_{s}} \Delta_{\mathrm{s}}^{\prime} .
\end{aligned}
$$

Using Eqs. (22), (42), (43), and (44) gives

$$
\begin{aligned}
\frac{1}{c} \frac{\partial \psi_{\mathrm{s}}}{\partial t} \cos \Theta & -\frac{\psi_{\mathrm{s}}}{c}\left[\frac{d \Delta \Theta}{d t}+\left.k_{\theta \mathrm{s}}\left(v_{\theta}+v_{* \mathrm{e}}\right)\right|_{r=r_{\mathrm{s}}}\right] \sin \Theta \\
= & -\nabla_{\| \text {in }}\left(\widetilde{\phi}+\frac{1}{e n_{0 \mathrm{~s}}} \widetilde{p}_{\mathrm{e}}+\frac{\alpha_{T}}{e} \widetilde{T}_{\mathrm{e}}\right)_{\text {in }}-\eta_{\|\|_{\| \text {in }}},
\end{aligned}
$$

with

$$
\begin{gathered}
v_{\theta}=\frac{c}{B_{0}} \widetilde{\phi}_{0}^{\prime}, \\
v_{* \mathrm{e}}=\frac{c}{B_{0}}\left(\frac{\widetilde{p}_{\mathrm{e} 0}^{\prime}}{e n_{0}}+\frac{\alpha_{T} \widetilde{T}_{\mathrm{e} 0}^{\prime}}{e}\right) .
\end{gathered}
$$

where $n_{0 \mathrm{~s}}=n_{0}\left(r_{\mathrm{s}}\right)$ and $\left\{\widetilde{\phi}_{0}, \widetilde{p}_{\mathrm{e} 0}, \widetilde{T}_{\mathrm{e} 0}\right\}$ are the perturbed equilibrium electrostatic potential, electron pressure, and electron temperature, respectively. Operating \langle\rangle$_{\Omega}$ to Eq. (71) gives

$$
\left.\frac{1}{c} \frac{\partial \psi_{\mathrm{s}}}{\partial t}\langle\cos \Theta\rangle_{\Omega}=-\eta_{\|} \widetilde{\tilde{j}_{\| \text {in }}}\right\rangle_{\Omega},
$$

where the constant- $\psi$ approximation and $\langle\sin \Theta\rangle_{\Omega}=0$ are used. Separating the inner layer current as

$$
\widetilde{j}_{\| \text {in }}=J_{0}(\Omega)+J_{1},
$$

where $J_{0}$ is the component constant on the $\Omega$ contour and $J_{1}$ is the residual component, Eq. (74) yields

$$
\widetilde{j}_{\| \text {in }}=-\frac{1}{\eta_{\|} c} \frac{\partial \psi_{\mathrm{s}}}{\partial t} \frac{\langle\cos \Theta\rangle_{\Omega}}{\langle 1\rangle_{\Omega}}+J_{1}-\frac{\left\langle J_{1}\right\rangle_{\Omega}}{\langle 1\rangle_{\Omega}} .
$$

Substituting Eq. (76) into Eq. (69), the cosine matching is written in the form of a modified Rutherford equation

$$
I_{1} \frac{\partial \psi_{\mathrm{s}}}{\partial t}=\frac{\eta_{\|} c^{2} \psi_{\mathrm{s}}}{2 \pi w} \Delta_{\mathrm{c}}^{\prime}+\eta_{\|} c I
$$

with

$$
\begin{gathered}
I_{1}=\sqrt{2} \int_{-1}^{\infty} d \Omega \frac{\langle\cos \Theta\rangle_{\Omega}^{2}}{\langle 1\rangle_{\Omega}} \\
I=\sqrt{2} \int_{-1}^{\infty} d \Omega\left[\left\langle J_{1} \cos \Theta\right\rangle_{\Omega}-\frac{\left\langle J_{1}\right\rangle_{\Omega}\langle\cos \Theta\rangle_{\Omega}}{\langle 1\rangle_{\Omega}}\right],
\end{gathered}
$$

where $I_{1} \approx 0.827^{2}$ and $J_{1}$ is specified in Subsection III D.

Substituting $\widetilde{j}_{\| \text {in }}$ in Eq. (71) into Eq. (70), we obtain an island phase evolution equation

$$
\begin{aligned}
& I_{2}\left[\frac{\partial \Delta \Theta}{\partial t}+\left.k_{\theta \mathrm{s}}\left(v_{\theta}+v_{* \mathrm{e}}\right)\right|_{r=r_{\mathrm{s}}}\right]=\frac{\sigma_{s} \eta_{\|} c^{2}}{w} \Delta_{\mathrm{s}}^{\prime} \\
& -\frac{4 c L_{s}}{k_{\theta \mathrm{s}} \psi_{\mathrm{s}} w} \int_{-1}^{\infty} d \Omega \oint_{\Omega} d \Theta\left[\nabla_{\| \mathrm{in}}^{2}\left(\widetilde{\phi}+\frac{\widetilde{p}_{\mathrm{e}}}{e n_{0 \mathrm{~s}}}+\frac{\alpha_{T} \widetilde{T}_{\mathrm{e}}}{e}\right)_{\mathrm{in}}\right]
\end{aligned}
$$

with

$$
I_{2}=\sqrt{2} \int_{-1}^{\infty} d \Omega \oint_{\Omega} d \Theta \sigma_{x} \sqrt{\Omega-\sigma_{s} \cos \Theta} \cos \Theta .
$$

In Eq. (80), $I_{2}$ diverges as $\Omega^{3 / 2}$ for $\Omega \rightarrow \infty$. The divergence of $I_{2}$ implies that the relation

$$
\frac{\partial \Delta \Theta}{\partial t}+\left.k_{\theta \mathrm{s}}\left(v_{\theta}+v_{* \mathrm{e}}\right)\right|_{r=r_{\mathrm{s}}}=0
$$

might be satisfied. More precisely, this anticipation is justified since the ideal MHD equations imply that $\nabla_{\| \text {in }}^{2}\left(\widetilde{\phi}+\widetilde{p}_{\mathrm{e}} / e n_{0 \mathrm{~s}}+\alpha_{T} \widetilde{T}_{\mathrm{e}} / e\right)_{\text {in }}$ is an odd function of $x$ in the large $\Omega$ regime, therefore, the right-hand side (RHS) of Eq. (80) might converge to a finite value.

Substituting $\nabla_{\|} \widetilde{j}_{\|}$in Eq. (21) in the inner layer into Eq. (70) gives another version of the sine matching, which corresponds to a force (torque) balance equation

$$
\begin{aligned}
& \int_{-1}^{\infty} d \Omega \oint_{\Omega} d \Theta\left[\frac{c^{2}}{4 \pi v_{\mathrm{A}}^{2}} \frac{D_{\mathrm{i}}}{D t} \nabla_{\perp}^{2} \phi-\frac{2 c}{B_{0}} \hat{z} \cdot \overline{\boldsymbol{\kappa}} \times \nabla_{\perp} p\right. \\
& \left.\quad-\frac{c^{2} \mu}{4 \pi v_{A}^{2}} \nabla_{\perp}^{4} \phi-\frac{c}{B_{0}} \hat{z} \cdot \nabla_{\perp} \times \overline{\left\langle\nabla \cdot \Pi^{\mathrm{neo}}\right\rangle_{\mathrm{srf}}}\right]_{\mathrm{in}}=-\frac{c k_{\theta \mathrm{s}} \psi_{\mathrm{s}}}{4 L_{s}} \Delta_{s}^{\prime},
\end{aligned}
$$

where $\nabla_{\| \text {in }} j_{\| 0}$ vanishes. 


\section{Modified Rutherford equation}

Following Refs. 2, 47, and 48, the ion and electron pressure profile in the inner layer are assumed to be determined by the local heat balance

$$
\chi_{\| \alpha} \nabla_{\|}^{2} p_{\alpha}+\chi_{\perp \alpha} \nabla_{\|}^{2} p_{\alpha}=0
$$

for $\alpha=\mathrm{i}$, e. Equations (25) and (26) are well approximated by Eq. (84) when the perpendicular thermal transport is dominated by the anomalous effect, the parallel thermal transport is much faster than any other parallel dynamics in the island region and the density perturbation is negligible. A typical scale length of Eq. (84) is $w_{\mathrm{c}, \alpha}=\left(\chi_{\perp \alpha} / \chi_{\| \alpha}\right)^{1 / 8}$ $\sqrt{8 L_{s} / k_{\theta s}}$. Then, Eq. (84) is written as ${ }^{47}$

$$
\begin{aligned}
& \left.\left.\frac{1}{4} \frac{w^{4}}{w_{\mathrm{c}, \alpha}^{4}} \frac{\partial}{\partial \Theta}\right|_{\Omega} \sqrt{\Omega-\sigma_{s} \cos \Theta} \frac{\partial}{\partial \Theta}\right|_{\Omega} p_{\alpha} \\
& +\left.\left.\frac{\partial}{\partial \Omega}\right|_{\Theta} \sqrt{\Omega-\sigma_{s} \cos \Theta} \frac{\partial}{\partial \Omega}\right|_{\Theta} p_{\alpha}=0,
\end{aligned}
$$

in the $(\Omega, \Theta)$-coordinates, and

$$
\frac{1}{4}\left(\left.\sigma_{s} X \frac{\partial}{\partial \Theta}\right|_{X}+\frac{w^{2}}{w_{\mathrm{c}, \alpha}^{2}} \sin \Theta \frac{\partial}{\partial X}\right)^{2} p_{\alpha}+\left.\frac{\partial^{2}}{\partial X^{2}}\right|_{\Theta} p_{\alpha}=0
$$

in the $(X, \Theta)$-coordinates, where $X=4 x / w_{\mathrm{c}, \alpha}$. In the limit of $w / w_{\mathrm{c}, \alpha} \gg 1$, the first term on LHS of Eq. (85) is dominant, and an approximate solution is

$$
\begin{gathered}
p_{\alpha}=\text { const. } \quad(-1 \leq \Omega \leq 1), \\
\left.\frac{\partial p_{\alpha}}{\partial \Omega}\right|_{\Theta}=\frac{\sqrt{2} \pi}{4} \frac{\sigma_{x} p_{\alpha 0 \mathrm{~s}}^{\prime} w}{\oint_{\Omega(x>0)} \sqrt{\Omega-\sigma_{s} \cos \Theta} d \Theta} \quad(1<\Omega),
\end{gathered}
$$

where $p_{\alpha 0 \mathrm{~s}}^{\prime}=p_{\alpha 0}^{\prime}\left(r_{\mathrm{s}}\right)$ and $p_{\alpha 0}$ is the unperturbed equilibrium pressure of the $\alpha$ species at the rational surface. In the limit of $w / w_{\mathrm{c}, \alpha} \ll 1$, a small parameter $w^{2} / w_{\mathrm{c}, \alpha}^{2}$ is used for an expansion parameter, and a perturbative solution of Eq. (86) is

$$
\begin{gathered}
p_{\alpha}=p_{\alpha 0 \mathrm{~s}}+p_{\alpha 0 \mathrm{~s}}^{\prime} x+p_{\alpha 1} \cos \Theta, \\
p_{\alpha 1}=\frac{\sigma_{s} p_{\alpha 0 \mathrm{~s}}^{\prime} w^{2} x}{16 x^{2}+\frac{w_{\mathrm{c}, \alpha}^{2}}{0.3}},
\end{gathered}
$$

which is a connecting version of Eqs. (37) and (38) in Ref. 47.

The influence of the polarization current, the anomalous viscosity, and the neoclassical viscosity on the island width evolution is typically smaller than that of the curvaturedriven current. Impacts of them will be discussed in Sec. III F. Further, the toroidal curvature is of order $O\left(r / R_{0}^{2}\right)$, which is typically smaller than $\kappa_{\mathrm{h}}$ by a factor of $l / M \ll 1$. Then, the parallel current perturbation is determined by a simplified version of Eq. (21)

$$
\nabla_{\| \text {in }} \widetilde{j}_{\| \text {in }}=-\frac{2 c}{B_{0}} \hat{\boldsymbol{z}} \cdot\left(\kappa_{\mathrm{hs}} \hat{\boldsymbol{x}}\right) \times\left(\nabla_{\perp} p\right)_{\mathrm{in}},
$$

which is rewritten as

$$
\left.\frac{\partial \widetilde{j}_{\| \text {in }}}{\partial \Theta}\right|_{\Omega}=\frac{2 \sigma_{s} c \kappa_{\mathrm{hs}} L_{s}}{m B_{0} x}\left(\left.\frac{\partial p}{\partial \theta}\right|_{x}\right)_{\text {in }},
$$

where a term $\nabla_{\| \text {in }} j_{\| 0}$ is neglected since it does not contribute to the inner-layer integral.

In the limit of $w \gg w_{\mathrm{c}, \alpha}$, substituting Eqs. (87) and (88) into Eq. (92) yields

$$
\begin{aligned}
& J_{1}=0 \quad(-1 \leq \Omega \leq 1), \\
& J_{1}=-\frac{4 \pi c \kappa_{\mathrm{hs}} L_{s} p_{0 \mathrm{~s}}^{\prime}}{B_{0}} \frac{\sigma_{x} \sqrt{\Omega-\sigma_{s} \cos \Theta}}{\oint_{\Omega(x>0)} \sqrt{\Omega-\sigma_{s} \cos \Theta} d \Theta} \quad(\Omega>1)
\end{aligned}
$$

where we have considered $\left.\left.\left.\partial_{\theta} p\right|_{x} \approx \partial_{\theta} \Omega\right|_{x} \partial_{\Omega} p\right|_{\Theta}$ in the region of $\Omega>1$. Substituting Eqs. (93) and (94) into Eq. (79) gives

$$
\begin{gathered}
I=I_{3} \frac{c \kappa_{\mathrm{hs}} L_{s} p_{0 \mathrm{~s}}^{\prime}}{4 B_{0}}, \\
I_{3}=16 \sqrt{2} \pi \int_{1}^{\infty} d \Omega \frac{\langle\cos \Theta\rangle_{\Omega}}{\langle 1\rangle_{\Omega} \oint_{\Omega, x>0} \sqrt{\Omega-\sigma_{s} \cos \Theta} d \Theta}
\end{gathered}
$$

where $I_{3} \approx 6.35 .^{4}$ In the limit of $w \ll w_{\mathrm{c}, \alpha}$, substituting Eqs. (89) and (90) into Eq. (92) yields

$$
J_{1}=\frac{c \kappa_{\mathrm{hs}} L_{s} p_{0 \mathrm{~s}}^{\prime}}{B_{0}} \frac{\cos \Theta}{\Omega+\frac{w_{\mathrm{c}, \alpha}^{2}}{0.6 w^{2}}},
$$

where we have considered $w_{\mathrm{c}, \alpha}^{2} / w^{2} \gg|\cos \Theta|$. Substituting Eq. (97) into Eq. (79) gives

$$
\begin{gathered}
I=\sum_{\alpha=\mathrm{i}, \mathrm{e}} I_{4}^{\alpha} \frac{c \kappa_{\mathrm{hs}} L_{s} p_{0 \mathrm{~s}}^{\prime}}{4 B_{0}} \frac{w}{w_{\mathrm{c}, \alpha}} \\
I_{4}^{\alpha}=4 \sqrt{2} \int_{-1}^{\infty} d \Omega \frac{1}{\Omega+\frac{w_{\mathrm{c}, \alpha}^{2}}{0.6 w^{2}}}\left(\left\langle\cos ^{2} \Theta\right\rangle_{\Omega}-\frac{\langle\cos \Theta\rangle_{\Omega}^{2}}{\langle 1\rangle_{\Omega}}\right),
\end{gathered}
$$

where $I_{4}$ weakly depends on $w_{\mathrm{c}, \alpha} / w$ but is typically $I_{4}^{\alpha} \approx 6.6$ for $1 \ll w_{\mathrm{c}, \alpha} / w \sim 10^{2}$.

Finally, the modified Rutherford equation is given by

$$
I_{1} \frac{4 \pi}{\eta_{\|} c^{2}} \frac{\partial w}{\partial t}=\Delta_{\mathrm{c}}^{\prime}+\sum_{\alpha=\mathrm{i}, \mathrm{e}} \frac{I_{3} D_{\alpha}}{\sqrt{w^{2}+\left(I_{3} / I_{4}\right)^{2} w_{\mathrm{c}, \alpha}^{2}}},
$$

with

$$
D_{\alpha}=\frac{8 \pi \kappa_{\mathrm{hs}} p_{\alpha 0 \mathrm{~s}}^{\prime} L_{s}^{2}}{B_{0}^{2}}
$$

where $I_{4}=6.6$. Note that $D=D_{\mathrm{i}}+D_{\mathrm{e}}$. The tokamak version of the third term on the RHS of Eq. (100) is first derived in Ref. 48, which formally agrees with our result except the detailed value of $I_{4}$. 


\section{E. Flow evolution equation}

In principle, Eq. (83) determines the flow evolution equation. However, to evaluate the integral in Eq. (83), $\phi$ and $p$ in the inner layer should be specified by some models. Since we only require perturbed equilibria of these variables, following Ref. 8, the integral in Eq. (83) is interpreted as an average inside magnetic islands.

Using Eqs. (21) and (40) for perturbed equilibria gives

$$
\begin{aligned}
\frac{c^{2}}{4 \pi v_{A}^{2}} \frac{\partial}{\partial t} \nabla_{\perp}^{2} \widetilde{\phi}_{0}= & -\frac{c^{2}}{4 \pi v_{A}^{2}}\left[\widetilde{\phi}-\frac{\widetilde{p}_{\mathrm{i}}}{e n_{0}}, \nabla_{\perp}^{2} \widetilde{\phi}\right]_{0,0}-\frac{1}{B_{0}}\left[A, \widetilde{j}_{\|}\right]_{0,0} \\
& +\frac{c^{2} \mu}{4 \pi v_{A}^{2}} \nabla_{\perp}^{4} \widetilde{\phi}_{0}+e \frac{1}{r} \frac{\partial}{\partial r} r\left(\Gamma_{\mathrm{i} 0}^{\text {neo }}-\Gamma_{\mathrm{e} 0}^{\text {neo }}\right),
\end{aligned}
$$

where the bracket $[,]_{0,0}$ indicates $(0,0)$ components of nonlinear couplings of Fourier modes and $\Gamma_{\alpha 0}^{\text {neo }}$ indicates the neoclassical particle flux of the $\alpha$ species in the perturbed equilibria. On the RHS of Eq. (102), the first term is the Reynolds stress and the second term is the so-called Maxwell stress. In the following, we neglect the Reynolds stress, since the Maxwell stress plays a dominant role in the presence of RMP. Considering that $[,]_{0,0}$ is interpreted by an averaging $\left(1 / 4 \pi^{2} R_{0}\right) \oint d \theta \oint d z$, the second term on the RHS of Eq. (102) operated by $-\left(B_{0} / r\right) \int_{0}^{r} d r r$ is expressed in the form of the $J \times B$ force $\left(\widetilde{j}_{\|} \widetilde{B}_{r}\right)_{0,0}$, where $\widetilde{B}_{r}=-\partial_{\theta} A$. The average $J \times B$ force near magnetic islands is

$$
\begin{aligned}
& \frac{1}{2 \pi w} \int_{-w / 2}^{w / 2} d x \int_{-\pi}^{\pi} d \Theta \frac{1}{c} \widetilde{j}_{\| \text {in }} \widetilde{B}_{r \text { in }} \approx \frac{k_{\theta \mathrm{s}} \psi_{s}}{8 \pi^{2} w} \int_{-\pi}^{\pi} d \Theta \sin \Theta\left[\frac{\partial A_{\mathrm{in}}}{\partial x}\right]_{-w / 2}^{+w / 2} \\
& \approx \frac{k_{\theta \mathrm{s}} B_{0}^{2}}{2048 \pi L_{s}^{2}} w^{3} \Delta_{\mathrm{s}}^{\prime}
\end{aligned}
$$

where $\widetilde{j}_{\| \text {in }}=-(c / 4 \pi) \partial_{x}^{2} A_{\text {in }}, \quad \widetilde{B}_{r \text { in }}=-\left(m / r_{s}\right) \psi_{s} \sin \Theta$, the constant- $\psi$ approximation, $\left[\partial A_{\text {in }} / \partial x\right]_{-w / 2}^{+w / 2}=\left[\partial A_{\text {out }} / \partial x\right]_{-w / 2}^{+w / 2}$ and Eq. (48) are used. Operating $-\left(c / r B_{0}\right) \int_{0}^{r} d r r$ to Eq. (102) and using Eq. (103), an evolution equation of the poloidal flow velocity is given by

$$
\begin{aligned}
\frac{\partial}{\partial t} v_{\theta}= & \sigma \frac{k_{\theta \mathrm{s}} v_{\mathrm{As}}^{2}}{512 L_{s}^{2}} w^{3} \Delta_{\mathrm{s}}^{\prime}+\mu \frac{\partial}{\partial r}\left[\frac{1}{r} \frac{\partial}{\partial r}\left(r v_{\theta}\right)\right] \\
& +\sum_{\alpha=\mathrm{i}, \mathrm{e}} \nu_{\alpha}^{\text {neo }}\left(V_{\alpha}^{\text {neo }}-v_{\theta}\right),
\end{aligned}
$$

with

$$
\begin{gathered}
\nu_{\alpha}^{\text {neo }}=\frac{15 \sqrt{\pi}}{8} \frac{\tau_{\alpha}}{\rho_{\mathrm{i}}^{2}} \frac{\epsilon_{\mathrm{t}} \epsilon_{\mathrm{h}}^{3 / 2} V_{\perp \alpha}^{2} \nu_{\alpha}}{c_{1} \epsilon_{\mathrm{t}} \epsilon_{\mathrm{h}}\left(v_{\theta} / r\right)^{2}+c_{2} \nu_{\alpha}^{2}}, \\
V_{\alpha}^{\text {neo }}=-\frac{c \widetilde{T}_{\alpha 0}}{e_{\alpha} B_{0}}\left(\frac{\widetilde{n}_{0}^{\prime}}{\widetilde{n}_{0}}+\frac{2 \widetilde{T}_{\alpha 0}^{\prime}}{\widetilde{T}_{\alpha 0}}\right),
\end{gathered}
$$

where $\left\{\widetilde{n}_{0}, \widetilde{T}_{\alpha 0}\right\}$ are the perturbed equilibrium density and temperature of the $\alpha$ species, respectively, $v_{\mathrm{As}}$ is the Alfven velocity at the rational surface, $\tau_{\mathrm{i}}=1, \tau_{\mathrm{e}}=T_{\mathrm{i}} / T_{\mathrm{e}}$, and $\sigma=1$ for $-w / 2 \leq x \leq w / 2$ and $\sigma=0$ for other cases. Considering the toroidal component of the $E \times B$ drift velocity, the toroidal flow velocity is given by

$$
v_{z}=\frac{r l}{R_{0}} v_{\theta} .
$$

Equation (104) is also written by the toroidal flow velocity using Eq. (107).

\section{F. Summary of model equations}

Collecting Eqs. (82), (100), and (104), the modified Rutherford equation, the island phase evolution equation and the poloidal flow evolution equation are

$$
\begin{aligned}
\frac{4 \pi I_{1}}{\eta_{\|} c^{2}} \frac{\partial w}{\partial t}=\Delta_{\text {mode }}^{\prime}+\Delta_{\text {coil }}^{\prime} \cos \Delta \Theta+\sum_{\alpha=\mathrm{i}, \mathrm{e}} \frac{I_{3} D_{\alpha}}{\sqrt{w^{2}+\left(I_{3} / I_{4}\right)^{2} w_{\mathrm{c}, \alpha}^{2}}} \\
\frac{\partial \Delta \Theta}{\partial t}=\left.k_{\theta \mathrm{s}}\left(v_{\theta}+v_{* \mathrm{e}}\right)\right|_{r=r_{\mathrm{s}}} \\
\frac{\partial v_{\theta}}{\partial t}=\sigma \frac{k_{\theta \mathrm{s}} v_{\mathrm{As}}^{2}}{512 L_{s}^{2}} w^{3} \Delta_{\text {coil }}^{\prime} \sin \Delta \Theta+\mu \frac{\partial}{\partial r}\left[\frac{1}{r} \frac{\partial}{\partial r}\left(r v_{\theta}\right)\right] \\
+\sum_{\alpha=\mathrm{i}, \mathrm{e}} \nu_{\alpha}^{\text {neo }}\left(V_{\alpha}^{\text {neo }}-v_{\theta}\right)
\end{aligned}
$$

where $\left\{\Delta_{\text {mode }}^{\prime}, \Delta_{\text {coil }}^{\prime}\right\}$ are calculated by Eqs. (53), (56), and (57), and $\left\{D_{\alpha}, \nu_{\alpha}^{\text {neo }}, V_{\alpha}^{\text {neo }}\right\}$ are given by Eqs. (101), (105), and (106), respectively.

The correction of the RHS of Eq. (108) due to the polarization current is roughly $\Delta_{\mathrm{pl}} \sim 8 \beta_{\mathrm{i}} \rho_{\mathrm{i}}^{2} L_{s}^{2} / r_{\mathrm{s}}^{2} w^{3}$, where $\beta_{\mathrm{i}}$ is the ion beta value. We have evaluated $\Delta_{\mathrm{pl}}$ for typical experimental parameters in LHD, and found that the influence is negligibly small in comparison with the other terms in Eq. (108). Similarly, the influence of the anomalous viscosity and the neoclassical viscosity on Eq. (108) is negligible. The toroidal direction of the equilibrium bootstrap current in stellarators is mainly in the opposite direction in comparison with that in tokamaks (so does the sign of the bootstrap current perturbation), ${ }^{45,49}$ therefore, the perturbed bootstrap current has the stabilizing effect on the island stability. As discussed in the Introduction, the influence of the perturbed bootstrap current does not play an essential role in low- $\beta$ stellarators. Therefore, these effects are neglected in our model for simplicity. However, the extension of the model might be required in an advanced parameter regime with high a $\beta$ value and a larger ion Larmor radius.

\section{CRITERION OF ISLAND STABILITY}

In the following, we consider that the ion temperature and the electron temperature in the equilibrium are comparable. In this case, the ion neoclassical viscosity dominates the electron neoclassical viscosity, and $\nu_{i}^{\text {neo }}$ is assumed to show the $1 / \nu_{\mathrm{i}}$ dependence, i.e., $\nu_{\mathrm{i}}^{\text {neo }} \approx \nu_{\mathrm{i} 0}^{\text {neo }}=\left.\nu_{\mathrm{i}}^{\text {neo }}\right|_{v_{\theta}=0}$. The poloidal flow profile outside magnetic islands is determined by the viscous force balance

$$
0=\mu \frac{\partial^{2} v_{\theta}}{\partial x^{2}}+\nu_{\mathrm{i} 0}^{\text {neo }}\left(V_{\mathrm{i}}^{\text {neo }}-v_{\theta}\right) .
$$

The solution of Eq. (111) is approximately given by $v_{\theta}-V_{\mathrm{i}}^{\text {neo }} \propto \exp (-|x| / \lambda)$, where 


$$
\lambda=\sqrt{\frac{\mu}{\nu_{\mathrm{i} 0}^{\text {neo }}}}
$$

is the typical scale length of flows. In the limit of $\lambda \gg w / 2$, the flow gradient is widely formed outside magnetic islands. While, in the limit of $\lambda \ll w / 2$, the gradient is sharply formed close to the separatrix. In both limits, the gradient is relaxed inside magnetic islands with the scale length $w / 2$. Therefore, Eq. (110) at the rational surface is reduced to a zero dimensional equation

$$
\begin{aligned}
\frac{\left.\partial v_{\theta}\right|_{r=r_{\mathrm{s}}}}{\partial t}= & \frac{k_{\theta \mathrm{s}} v_{\mathrm{As}}^{2} w^{3}}{512 L_{s}^{2}} \Delta_{\mathrm{s}}^{\prime}+\frac{2 \mu}{w d}\left(V_{\mathrm{i} 0}^{\text {neo }}-\left.v_{\theta}\right|_{r=r_{\mathrm{s}}}\right) \\
& +\nu_{\mathrm{i} 0}^{\text {neo }}\left(V_{\mathrm{is}}^{\text {neo }}-\left.v_{\theta}\right|_{r=r_{\mathrm{s}}}\right),
\end{aligned}
$$

with

$$
d=\left\{\begin{aligned}
\lambda & (\lambda \gg w / 2) \\
w & (\lambda \ll w / 2)
\end{aligned}\right.
$$

where $V_{\text {is }}^{\text {neo }}=V_{\mathrm{i}}^{\text {neo }}\left(r_{\mathrm{s}}\right)$ is the perturbed neoclassical flow velocity at the rational surface and $V_{\mathrm{i} 0}^{\text {neo }}$ is the unperturbed neoclassical flow velocity at the rational surface. It is easily confirmed that the second term dominates the third term on the RHS of Eq. (113) if $\lambda \ll w / 2$, and vice versa. Therefore, it is convenient to define

anomalous viscosity - dominant regime: $\lambda \gg w / 2$, neoclassical viscosity - dominant regime: $\lambda \ll w / 2$.

Then, simplified and normalized model equations are

$$
\begin{gathered}
S \frac{d \hat{w}}{d T}=-\frac{\Delta_{*}^{\prime}}{\Delta_{0}^{\prime}}+\frac{\hat{w}_{\mathrm{v}}^{2}}{\hat{w}^{2}} \cos \Delta \Theta+\frac{\hat{D}}{\hat{w}}, \\
\frac{d \Delta \Theta}{d T}=\hat{\omega}, \\
\frac{d v}{d T}=-M \hat{w} \hat{w}_{\mathrm{v}}^{2} \sin \Delta \Theta+\frac{\hat{\mu}}{\hat{w} \hat{d}}\left(\hat{V}_{0}-v\right)+\hat{\nu}\left(\hat{V}_{\mathrm{s}}-v\right),
\end{gathered}
$$

with

$$
\hat{d}= \begin{cases}\hat{\lambda} & (\hat{\lambda} \gg \hat{w} / 2) \\ \hat{w} & (\hat{\lambda} \ll \hat{w} / 2)\end{cases}
$$

where variables are normalized as $\hat{w}=w / r_{\mathrm{s}}, \hat{w}_{\mathrm{v}}=w_{\mathrm{v}} / r_{\mathrm{s}}$, $\hat{\lambda}=\lambda / r_{\mathrm{s}}$, and $T=t / \tau_{\mathrm{A}}$, and parameters are defined by $\tau_{\mathrm{A}}=r_{\mathrm{s}} / v_{\mathrm{A}}, v=\left.v_{\theta}\right|_{r_{\mathrm{s}}} / v_{\mathrm{A}}, \hat{\mu}=2 \mu\left(\tau_{\mathrm{A}} / r_{\mathrm{s}}^{2}\right), \hat{V}_{0}=V_{\mathrm{i} 0}^{\mathrm{nc}} / v_{\mathrm{A}}, \hat{V}_{\mathrm{s}}$ $=V_{\mathrm{is}}^{\mathrm{nc}} / v_{\mathrm{A}}, \hat{\omega}=\omega \tau_{\mathrm{A}}, \omega=k_{\theta \mathrm{s}}\left(V_{\mathrm{is}}^{\text {neo }}+v_{* \mathrm{e}}\left(r_{\mathrm{s}}\right)\right), \hat{\nu}=\nu_{\mathrm{i} 0}^{\text {neo }} \tau_{\mathrm{A}}$, $S=I_{1}\left(\tau_{\mathrm{R}} / \tau_{\mathrm{A}}\right) /\left(r_{\mathrm{s}} \Delta_{0}^{\prime}\right)$, and $M=\left(-\Delta_{0}^{\prime} k_{\theta \mathrm{s}} r_{\mathrm{s}}^{4}\right) /\left(512 L_{s}^{2}\right)$. Here, $\hat{D}$ and $\Delta_{*}^{\prime}$ include the curvature effects, and we will specify these parameters in the following subsections.

\section{A. Self-healing threshold}

To evaluate a criterion of the self-healing, i.e., the spontaneous shrinkage of locked magnetic islands, we assume that the following conditions are satisfied: $w \sim w_{\mathrm{c}, \mathrm{i}}>w_{\mathrm{c}, \mathrm{e}}$, $\hat{\omega}=0, v=0$. The model equations for large magnetic islands in the steady state are reduced to

$$
\begin{gathered}
0=-\frac{\Delta_{* 1}^{\prime}}{\Delta_{0}^{\prime}}+\frac{\hat{w}_{\mathrm{v}}^{2}}{\hat{w}^{2}} \cos \Delta \Theta+\frac{\hat{D}_{1}}{\hat{w}}, \\
0=-M \hat{w} \hat{w}_{\mathrm{v}}^{2} \sin \Delta \Theta+\frac{\hat{\mu}}{\hat{w} \hat{d}} \hat{V}_{0}+\hat{\nu} \hat{V}_{\mathrm{s}},
\end{gathered}
$$

with

$$
\begin{gathered}
\Delta_{* 1}^{\prime}=\Delta_{0}^{\prime}, \\
\hat{D}_{1}=\frac{\left(2+I_{3}\right) D_{\mathrm{e}}}{\left(-\Delta_{0}^{\prime} r_{\mathrm{s}}\right)},
\end{gathered}
$$

where we have considered Eqs. (58) and (61). Using Eqs. (119) and (120), we obtain

$$
F(\hat{w})=\frac{\hat{w}^{8}}{\hat{w}_{\mathrm{v}}^{4}}\left(1-\frac{\hat{D}_{1}}{\hat{w}}\right)+\frac{1}{M^{2} \hat{w}_{\mathrm{v}}^{4}}\left(\frac{\hat{\mu} \hat{w}}{\hat{d}} \hat{V}_{0}+\hat{\nu} \hat{V}_{\mathrm{s}} \hat{w}^{2}\right)-\hat{w}^{4}=0 .
$$

At the critical value of $\hat{w}_{\mathrm{v}}$, below which magnetic islands start to rotate and shrink, the condition $d F / d \hat{w}=0$ is also satisfied since the minimal value of $\hat{w}_{\mathrm{v}}$ is on the $\hat{w}_{\mathrm{v}}$-axis. To approximately solve $F=0$ and $d F / d \hat{w}=0, \hat{D}_{1}$ is used for a small parameter, and the variables are expanded as $\hat{w}=\hat{w}_{0}+\hat{w}_{1}, \Delta \Theta=\Delta \Theta_{0}+\Delta \Theta_{1}$, and $\hat{w}_{\mathrm{v}}=\hat{w}_{\mathrm{v} 0}+\hat{w}_{\mathrm{v} 1}$.

In the anomalous viscosity-dominant regime, where the fourth term on the RHS of Eq. (123) is negligible, solutions are given by $\hat{w}_{0}=\hat{w}_{\mathrm{v} 0} / 2^{1 / 4}, \hat{w}_{1}=3 \hat{D}_{1} / 16, \Delta \Theta_{0}=\pi / 4$, $\Delta \Theta_{1}=2 \hat{w}_{1} / 3 \hat{w}_{0}, \hat{w}_{\mathrm{v} 0}=2^{1 / 4}\left(\hat{\mu} \hat{\nu} \hat{V}_{0}^{2} / M^{2}\right)^{1 / 8}$, and $\hat{w}_{\mathrm{v} 1}=-2^{1 / 4}$ $\hat{D}_{1} / 4$. The self-healing criterion is written by the RMP amplitude at the edge boundary $B_{\mathrm{RMP}}=k_{\theta \mathrm{a}} \psi_{a} / a$, where $k_{\theta \mathrm{a}}=m / a$, such that

$$
\begin{aligned}
& \frac{B_{\mathrm{RMP}}^{\text {heal }}}{B_{0}}=\frac{B_{\mathrm{RMP} 0}^{\text {heal }}}{B_{0}}+\frac{B_{\mathrm{RMP} 1}^{\mathrm{heal}}}{B_{0}}, \\
& \frac{B_{\mathrm{RMP} 0}^{\text {heal }}}{B_{0}}=\alpha_{1} L\left(\frac{\hat{\mu} \hat{\nu} \hat{V}_{0}^{2}}{M^{2}}\right)^{1 / 4},
\end{aligned}
$$

$$
\frac{B_{\mathrm{RMP} 1}^{\mathrm{heal}}}{B_{0}}=-\alpha_{2} L \hat{D}_{1}\left(\frac{\hat{\mu} \hat{\nu} \hat{V}_{0}^{2}}{M^{2}}\right)^{1 / 8}
$$

where $L=(1 / 16)\left(k_{\theta a} r_{\mathrm{s}}^{2} / L_{s}\right)\left(a / r_{\mathrm{s}}\right)^{m}, \alpha_{1}=2^{1 / 2}, \alpha_{2}=2^{-1 / 2}$.

In the neoclassical viscosity-dominant regime, where the third term on the RHS of Eq. (123) is negligible, solutions are $\hat{w}_{0}=\hat{w}_{v 0} / 3^{1 / 4}, \hat{w}_{1}=\hat{D}_{1} / 4, \Delta \Theta_{0}=\cos ^{-1}(1 / \sqrt{3})$, $\Delta \Theta_{1}=2^{1 / 2} \hat{w}_{1} / 3 \hat{w}_{0}, \quad \hat{w}_{v 0}=\left(3^{1 / 4} / 2^{1 / 6}\right)\left(\hat{\nu} \hat{V}_{\mathrm{s}} / M\right)^{1 / 4}, \quad$ and $\hat{w}_{\mathrm{v} 1}=-3^{1 / 4} \hat{D}_{1} / 6$. Again, the self-healing criterion is written by the RMP amplitude such that

$$
\begin{aligned}
& \frac{B_{\mathrm{RMP}}^{\mathrm{heal}}}{B_{0}}=\frac{B_{\mathrm{RMP} 0}^{\mathrm{heal}}}{B_{0}}+\frac{B_{\mathrm{RMP} 1}^{\mathrm{heal}}}{B_{0}}, \\
& \frac{B_{\mathrm{RMP} 0}^{\text {heal }}}{B_{0}}=\alpha_{3} L\left(\frac{\hat{\nu} \hat{V}_{\mathrm{s}}}{M}\right)^{2 / 3},
\end{aligned}
$$




$$
\frac{B_{\mathrm{RMP} 1}^{\mathrm{heal}}}{B_{0}}=-\alpha_{4} \hat{D}_{1} L\left(\frac{\hat{\nu} \hat{V}_{\mathrm{s}}}{M}\right)^{1 / 3},
$$

where $\alpha_{3}=2^{-1 / 3} 3^{1 / 2}$ and $\alpha_{4}=2^{-1 / 6} 3^{-3 / 4}$.

Above results clearly show that the self-healing threshold is shifted by the curvature effect.

\section{B. Penetration threshold}

To evaluate a criterion of the penetration RMP, i.e., the back transition of the self-healing, we assume $w \sim w_{\text {c.e }}$ $<w_{\mathrm{c}, \mathrm{i}}, \quad \hat{\omega}=\hat{\omega}_{0}+k v, \quad \hat{V}_{\mathrm{s}}=\hat{V}_{0}, \quad$ where $\hat{\omega}_{0}=\tau_{\mathrm{A}} k_{\theta \mathrm{s}}\left(V_{\mathrm{i} 0}^{\text {neo }}\right.$ $\left.+v_{* \mathrm{e} 0}\right), k=k_{\theta \mathrm{s}} r_{\mathrm{s}}=m$, and $v_{* \mathrm{e} 0}$ is the unperturbed electron diamagnetic drift velocity. The model equations for healed magnetic islands are reduced to

$$
\begin{gathered}
S \frac{d \hat{w}}{d T}=-\frac{\Delta_{* 2}^{\prime}}{\Delta_{0}^{\prime}}+\frac{\hat{w}_{\mathrm{v}}^{2}}{\hat{w}^{2}} \cos \Delta \Theta+\frac{\hat{D}_{2}}{\hat{w}}, \\
\frac{d \Delta \Theta}{d T}=\hat{\omega}_{0}+k\left(v-\hat{V}_{0}\right), \\
\frac{d v}{d T}=-M \hat{w} \hat{w}_{\mathrm{v}}^{2} \sin \Delta \Theta+\left(\frac{\hat{\mu}}{\hat{w} \hat{d}}+\hat{\nu}\right)\left(\hat{V}_{0}-v\right) .
\end{gathered}
$$

with

$$
\begin{gathered}
\Delta_{* 2}^{\prime}=\Delta_{0}^{\prime}+I_{4}\left(\frac{D_{\mathrm{e}}}{w_{\mathrm{c}, \mathrm{e}}}+\frac{D_{\mathrm{i}}}{w_{\mathrm{c}, \mathrm{i}}}\right), \\
\hat{D}_{2}=\frac{2 D}{\left(-\Delta_{0}^{\prime} r_{\mathrm{s}}\right)},
\end{gathered}
$$

where we remind $D=D_{\mathrm{e}}+D_{\mathrm{i}}$. If the RMP amplitude is small enough, Eqs. (130)-(132) describe small oscillating (rotating) islands with unlocked flows. When the RMP amplitude reaches a critical value, the $J \times B$ force starts to damp flows, which slows down the island rotation and triggers the sudden island growth. To evaluate the criterion, we consider that the time average of $v$ is $\hat{V}_{0}$, then the time-average island phase evolves as $\hat{\omega}_{0} T$. If magnetic islands are stable in the absence of the RMP even for the island width close to the linearlayer width $\delta_{\text {lin }}$, i.e., $\Delta_{*}^{\prime} / \Delta_{0}^{\prime}+\hat{D}_{2} / \delta_{\text {lin }}<0$, the second term on the RHS of Eq. (130) is dominant for the island evolution, and islands approximately evolves as

$$
\hat{w}=\hat{w}_{\omega}\left|\sin \left(\omega_{0} T\right)\right|^{1 / 3},
$$

where $\hat{w}_{\omega}=\left(3 \hat{w}_{v}^{2} / S \hat{\omega}_{0}\right)^{1 / 3}$

In contrast, if magnetic islands are unstable even in the absence of RMP, the time-average island width becomes

$$
\hat{w}_{D}=\frac{\hat{D}_{2} \Delta_{0}^{\prime}}{\Delta_{* 2}^{\prime}} .
$$

In the following, the force balance is discussed in the different viscosity regime. First, we consider the anomalous viscosity-dominant regime. The locking of flows is triggered when the maximum $J \times B$ force overcomes the maximum viscous force. Substituting Eq. (135) into Eq. (132) gives the penetration threshold

$$
\frac{B_{\mathrm{RMP} 0}^{\mathrm{pen}}}{B_{0}}=\alpha_{5} L\left(S \hat{\omega}_{0}\right)^{2 / 5}\left(\frac{\hat{\mu} \hat{\nu} \hat{V}_{0}^{2}}{M^{2}}\right)^{3 / 10}
$$

where $\alpha_{5}=3^{-2 / 5}$ and the subscript " 0 " of $B_{\mathrm{RMP} 0}^{\mathrm{pen}}$ indicates that the threshold is reproduced in the case of $D=0$. Similarly, substituting Eq. (136) into Eq. (132) gives

$$
\frac{B_{\mathrm{RMP}}^{\mathrm{pen}}}{B_{0}}=L\left(\frac{\Delta_{* 2}^{\prime}}{\hat{D}_{2} \Delta_{0}^{\prime}}\right)^{2}\left(\frac{\hat{\mu} \hat{\nu} \hat{V}_{0}^{2}}{M^{2}}\right)^{1 / 2} .
$$

Next, we consider the neoclassical viscosity-dominant regime. Substituting Eq. (135) into Eq. (132) gives

$$
\frac{B_{\mathrm{RMP} 0}^{\mathrm{pen}}}{B_{0}}=\alpha_{6} L\left(S \hat{\omega}_{0}\right)^{1 / 4}\left(\frac{\hat{\nu} \hat{V}_{0}}{M}\right)^{3 / 4},
$$

where $\alpha_{6}=3^{-1 / 4}$. Finally, substituting Eq. (136) into Eq. (132) gives

$$
\frac{B_{\mathrm{RMP}}^{\text {pen }}}{B_{0}}=\frac{L \Delta_{* 2}^{\prime}}{\hat{D}_{2}\left(-\Delta_{0}^{\prime}\right)} \frac{\hat{\nu} \hat{V}_{0}}{M} .
$$

It is remarkable that the penetration threshold is essentially modified when magnetic islands are nonlinearly destabilized by the curvature effect. Note that such modification is effective when $\hat{w}_{D}$ overcomes $\hat{w}_{\omega}$.

\section{Hysteresis characteristics}

Figure 1 shows the schematic stability diagram of RMPinduced magnetic islands in the space of $D$ and $B_{\mathrm{RMP}}$, using the various thresholds derived in Subsections IV A and IV B, where $D_{\mathrm{e}}=D_{\mathrm{i}}=D / 2$ is assumed for simplicity. The diagram is almost the same for the anomalous viscositydominant regime and the neoclassical viscosity-dominant regime. In Fig. $1, D=D_{1}$ is determined by $\hat{w}_{D}=\hat{w}_{\omega}$. In Fig. 1 , hysteresis characteristics of the island state is clearly observed, i.e., once locked magnetic islands are healed, sufficiently larger RMP amplitude is necessary to excite locked magnetic islands. In opposite, the self-healing does not take place even below the penetration threshold. In the presence of the strong curvature effect, the self-healing tends not to take place, while the penetration tends to occur.

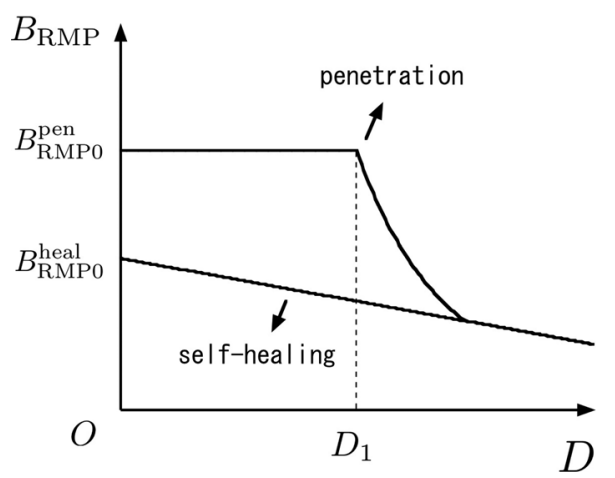

FIG. 1. Schematic diagram of nonlinear states of magnetic islands regime in a $\left(D, B_{\mathrm{RMP}}\right)$ space. 
In the following, to evaluate the magnitude for the hysteresis, we only consider the low- $\beta$ limit in the following, where the curvature effect is negligible. In the anomalous viscositydominant regime, coupling Eq. (125) to Eq. (137) gives

$$
\frac{B_{\mathrm{RMP} 0}^{\mathrm{pen}}}{B_{0}}=\alpha_{7}\left(\frac{S^{2} \hat{\omega}_{0}^{2}}{L}\right)^{1 / 5}\left(\frac{B_{\mathrm{RMP} 0}^{\mathrm{heal}}}{B_{0}}\right)^{6 / 5},
$$

where $\alpha_{7}=\alpha_{1}^{-6 / 5} \alpha_{5}$, whereas, in the neoclassical viscositydominant regime, coupling Eq. (128) to Eq. (139) gives

$$
\frac{B_{\mathrm{RMP} 0}^{\mathrm{pen}}}{B_{0}}=\alpha_{8}\left(\frac{\hat{V}_{0}}{\hat{V}_{\mathrm{s}}}\right)^{3 / 4}\left(\frac{S^{2} \hat{\omega}_{0}^{2}}{L}\right)^{1 / 8}\left(\frac{B_{\mathrm{RMP} 0}^{\mathrm{heal}}}{B_{0}}\right)^{9 / 8},
$$

where $\alpha_{8}=\alpha_{3}^{-9 / 8} \alpha_{6}$. Note that $\hat{V}_{0} / \hat{V}_{\mathrm{s}} \geq 1$. If the factor $S^{2} \hat{\omega}_{0}^{2} / L$ is much larger than unity, $B_{\mathrm{RMP} 0}^{\mathrm{pen}}$ is larger than $B_{\mathrm{RMP} 0}^{\text {heal }}$. It is remarkable that $S^{2} \hat{\omega}_{0}^{2} / L$ is independent from the detailed modeling of the viscosity. Here, we assume $T_{\mathrm{e}}=T_{\mathrm{i}}$, scale lengths of the density and the temperature gradients are of order $r_{\mathrm{s}}, \Delta_{0}^{\prime} \approx-2 k_{\theta \mathrm{s}}, r_{\mathrm{s}} / a$ is of order unity, $\hat{V}_{\text {is }}^{\text {neo }} \approx \hat{V}_{\mathrm{i} 0}^{\text {neo }}$ and the anomalous viscosity coefficient is approximated by the Bohm-type diffusion coefficient $\left(f_{\mu} / 16\right)\left(c T_{\mathrm{i}} / e B_{0}\right)$ or gyro-Bohm-type diffusion coefficient $\left(f_{\mu} / 16\right)\left(\rho_{\mathrm{i}} / r_{\mathrm{s}}\right)\left(c T_{\mathrm{i}}\right.$ $\left./ e B_{0}\right)$, where $f_{\mu}$ is a fitting parameter. We introduce dimensionless parameters: the normalized ion collisionality $\nu_{*}$ $=\nu_{\mathrm{i}}\left(r_{\mathrm{s}} / v_{\mathrm{ti}}\right)$, the normalized ion Larmor radius $\rho_{*}=\rho_{\mathrm{i}} / r_{\mathrm{s}}$, and the normalized ion skin depth $\delta=c /\left(\omega_{\mathrm{pi}} r_{\mathrm{s}}\right)$, where $v_{\mathrm{ti}}$ is the ion thermal velocity and $\omega_{\mathrm{pi}}$ is the ion plasma oscillation frequency. Each parameter is measured at the rational surface. The index of the hysteresis scales as

$$
\frac{S^{2} \hat{\omega}_{0}^{2}}{L}=\gamma_{1} \epsilon_{\mathrm{t}} \nu_{*}^{-1 / 2} \rho_{*}^{2} s^{-1} \delta^{-4},
$$

where $\gamma_{1}=0.0229 \times n^{-1} A$ and $A$ is the mass number of the hydrogen. The self-healing and penetration thresholds of the RMP amplitude in the anomalous viscosity-dominant regime are rewritten as

$$
\begin{gathered}
\frac{B_{\mathrm{RMP} 0}^{\text {heal }}}{B_{0}}=\gamma_{2} \epsilon_{\mathrm{t}}^{1 / 4} \epsilon_{\mathrm{h}}^{3 / 8} \beta_{\mathrm{i}}^{1 / 2} \nu_{*}^{-1 / 4} \rho_{*}^{k / 4+3 / 4} s^{0}, \\
\frac{B_{\mathrm{RMP} 0}^{\text {pen }}}{B_{0}}=\gamma_{3} \epsilon_{\mathrm{t}}^{1 / 10} \epsilon_{\mathrm{h}}^{1 / 20} \beta_{\mathrm{i}}^{9 / 20} \nu_{*}^{-7 / 10} \rho_{*}^{3 k / 10+1} s^{-1 / 5} \delta^{-1 / 5},
\end{gathered}
$$

where $\gamma_{2}=1.06 \times f_{\mu}^{1 / 4}, \quad \gamma_{3}=0.170 \times n^{-1 / 5} A^{1 / 5} f_{\mu}^{3 / 10}$, and $k=0(k=1)$ indicates the Bohm-type (gyro-Bohm-type) modeling of the anomalous viscosity. Whereas, those in the neoclassical viscosity-dominant regime are rewritten as

$$
\begin{array}{r}
\frac{B_{\mathrm{RMP} 0}^{\text {heal }}}{B_{0}}=\gamma_{4} \epsilon_{\mathrm{t}} \epsilon_{\mathrm{h}} \beta_{\mathrm{i}}^{2 / 3} \nu_{*}^{-2 / 3} \rho_{*}^{2 / 3} s^{-1 / 3}, \\
\frac{B_{\mathrm{RMP} 0}^{\text {pen }}}{B_{0}}=\gamma_{5} \epsilon_{\mathrm{t}}^{1 / 4} \epsilon_{\mathrm{h}}^{9 / 8} \beta_{\mathrm{i}}^{3 / 4} \nu_{*}^{-1} \rho_{*} s^{-1 / 2} \delta^{-1 / 2},
\end{array}
$$

where $\gamma_{4}=4.86 \times n^{-1 / 3}$ and $\gamma_{5}=1.96 \times n^{-1 / 2} A^{1 / 8}$. Criteria of arbitrary dimensionless parameters for the self-healing and penetration for the fixed RMP amplitude are easily obtained by solving the Eqs. (144)-(147) for the requiring parameters.
Figure 2 shows the stability diagram of RMP-induced magnetic islands in the space of $\beta_{\mathrm{i}}$ and $\nu_{*}$, using Eqs. (144)(147). The transition from the anomalous viscosity-dominant regime to the neoclassical viscosity-dominant regime occurs at $\hat{\lambda}=\hat{w} / 2$. For the suppressed islands, $\hat{w}=\left(3 \hat{w}_{v}^{2} / S \hat{\omega}_{0}\right)^{1 / 3}$ and $\hat{\lambda}=\hat{w} / 2$ give the transition point $\nu_{* 1}=2.46$ $\times 10^{6} n^{-2} A^{-1} f_{\mu}^{-3 k} \epsilon_{t} \epsilon_{h}^{9 / 2} \rho_{*}^{-3 k-5} s^{-2} \hat{\delta}^{4}\left(B_{\mathrm{RMP}} / B_{0}\right)^{2}$. Whereas, for locked magnetic islands comparable to the vacuum islands, $\hat{w}=\hat{w}_{\mathrm{v}}$ and $\hat{\lambda}=\hat{w} / 2$ give the transition point $\nu_{* 2}=5 \sqrt{\pi} f_{\mu}^{-k} \hat{w}_{\mathrm{v}} \rho_{*}^{-k-1}$. Here, $\beta_{\mathrm{i} 1}\left(\beta_{\mathrm{i} 2}\right)$ is evaluated by substituting $\nu_{* 1}\left(\nu_{* 2}\right)$ into Eq. (145) (Eq. (144)) for the fixed RMP amplitude and solving for $\beta_{\mathrm{i}}$. In Fig. 2, hysteresis characteristics of the island state and the change of $\beta$ dependency are observed. In the small $\nu_{*}$ regime, the $\beta$ dependence is due to the direct influence of the neoclassical viscosity, while, in the large $\nu_{*}$ regime, which is due to the anomalous viscosity coupling to the neoclassical viscosity, as shown in Eqs. (120) and (132). In particular, the $\beta$ dependence for the selfhealing threshold is strongly enhanced in large $\beta$ regime.

\section{NUMERICAL ANALYSIS}

Here, Eqs. (53), (56), (57), (101), (108), (109), and (110) are numerically solved for the typical parameters in the LHD: $\quad R_{0}=3.6[\mathrm{~m}], \quad a=0.7[\mathrm{~m}], \quad l=2, \quad M=10$, $B_{0}=1.5[\mathrm{~T}], \epsilon_{\mathrm{h}}=\epsilon_{\mathrm{ha}}(r / a)^{l}, \epsilon_{\mathrm{ha}}=0.4, \quad l=0.4+1.2(r / a)^{4}$, $n_{0}=2 \times 10^{-19}\left[\mathrm{~m}^{-3}\right], T_{\mathrm{i} 0}=T_{\mathrm{e} 0}=T_{0}\left[1-(r / a)^{2}\right]$ with $T_{0}=$ $1[\mathrm{keV}]$ and $\beta_{\mathrm{s}}=0.10[\%]$, where $\beta_{\mathrm{s}}$ is the $\beta$ value at the rational surface. The boundary condition of the poloidal flow velocity is $v_{\theta}(r=0)=v_{\theta}(r=a)=0$. In our parameters, the ion neoclassical viscosity dominates the electron neoclassical viscosity in Eq. (110), i.e., $\nu_{\mathrm{i}}^{\text {neo }} \gg \nu_{\mathrm{e}}^{\text {neo }}$, and poloidal flows are in the direction of the ion neoclassical flows. ${ }^{50}$ Moreover, $\nu_{\mathrm{i}}^{\text {neo }}$ shows the $1 / \nu_{\mathrm{i}}$ dependence, which is consistent with the assumption made in Sec. IV. The rational surface $l_{\mathrm{s}}=1$ is located at $r_{\mathrm{s}} / a=0.84$ and magnetic islands and RMP have mode numbers $(m, n)=(1,1)$. The anomalous diffusivities are $\chi_{\perp}^{\mathrm{e}}=\chi_{\perp}^{\mathrm{i}}=3\left[\mathrm{~m}^{2} / \mathrm{s}\right]$, which are typical values in the experiments in LHD. ${ }^{19}$ For these parameters, $a \Delta_{0}^{\prime}=-8.2$, the tearing mode is in the visco-resistive regime, ${ }^{8}$ where the visco-resistive linear-layer width is given by $\delta_{\mathrm{VR}} / a=6.3 \times 10^{-3}, w_{\mathrm{c}, \mathrm{i}}=6.8 \times 10^{-2}$, and $w_{\mathrm{c}, \mathrm{e}}=3.1$ $\times 10^{-2}$. To evaluate the perturbed equilibrium temperature $T_{\alpha 0}$, the local heat balance near magnetic islands Eq. (84)

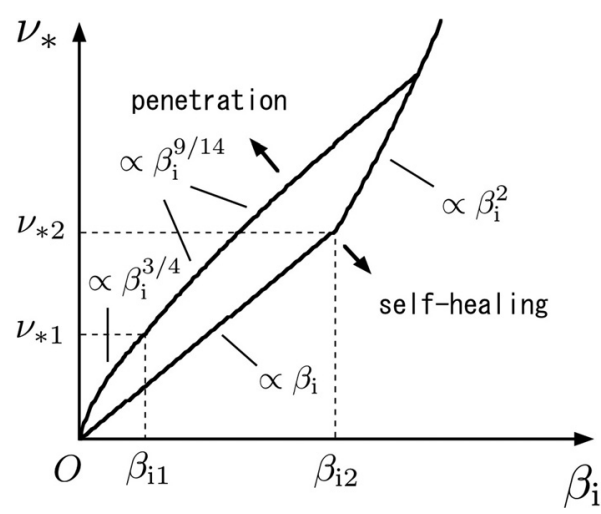

FIG. 2. Schematic diagram of nonlinear states magnetic islands in a $\left(\beta_{\mathrm{i}}, \nu_{*}\right)$, where $D=0$ is considered as a typical low- $\beta$ case. 
should be solved, however, this is quite time-consuming. The solution equations (87) and (89) indicate that the temperature profile of the $\alpha$ species is flattened near magnetic islands if $w \gg w_{\mathrm{c} \alpha}$. Therefore, we consider

$$
\widetilde{T}_{\alpha 0}=T_{\alpha 0}-\frac{T_{\alpha 0 \mathrm{~s}}^{\prime} x}{1+(2 x / w)^{p_{1}}} \frac{w^{p_{2}}}{2 w^{p_{2}}+w_{\mathrm{c} \alpha}^{p_{2}}},
$$

for $\alpha=\mathrm{i}$, e, where $T_{\alpha 0 \mathrm{~s}}^{\prime}=T_{\alpha 0}^{\prime}\left(r_{\mathrm{s}}\right), p_{1}=4$, and $p_{2}=2$ are chosen. The anomalous viscosity and the RMP amplitude are used for parameter scan. We set $w=\delta_{\mathrm{VR}}$ in case of $w<\delta_{\mathrm{VR}}$, since the nonlinear theory is applicable to cases with $w>\delta_{\mathrm{VR}}$.

First, we examine simulations with ramp-up/down RMP amplitudes. In the ramp-up phase, $B_{\mathrm{RMP}} / B_{0}$ is linearly increased from zero to $10^{-3}$, then, in the ramp-down phase, $B_{\mathrm{RMP}} / B_{0}$ is linearly decreased from $10^{-3}$ to zero, where the total time of the change in $B_{\mathrm{RMP}} / B_{0}$ is $10[\mathrm{~s}]$ for each phase. Since $10[\mathrm{~s}]$ is sufficiently longer than the time scale of the island growth and rotation, results might reproduce saturation states in many simulations with fixed RMP amplitudes.

Figure 3 shows the RMP amplitude dependence of the magnetic island width. In the early ramp-up phase, magnetic islands are rotating and the island growth is suppressed by the time-periodical phase shift between the islands and vacuum islands. In the suppressed state, the magnetic island width rapidly grows and damps to the linear-layer width, and this cycle is repeated as described in Eq. (135). When the RMP amplitude reaches a sufficiently large value, the penetration suddenly occurs and large locked islands comparable to vacuum islands are excited. In the present parameters, the curvature parameter is $D=0.028$, which is small to excite the curvature-driven magnetic islands. Therefore, the curvature hardly affects the suppressed state and the penetration threshold. In the early ramp-down phase, large locked islands are maintained. When the RMP amplitude becomes sufficiently small, the viscous force overcomes the $J \times B$ locking force, and the locking of poloidal flows is not sustained. Then, islands start to rotate and transit to the suppressed state, which is the self-healing of islands by poloidal flows. Extended view of Fig. 3 is shown is Fig. 4.

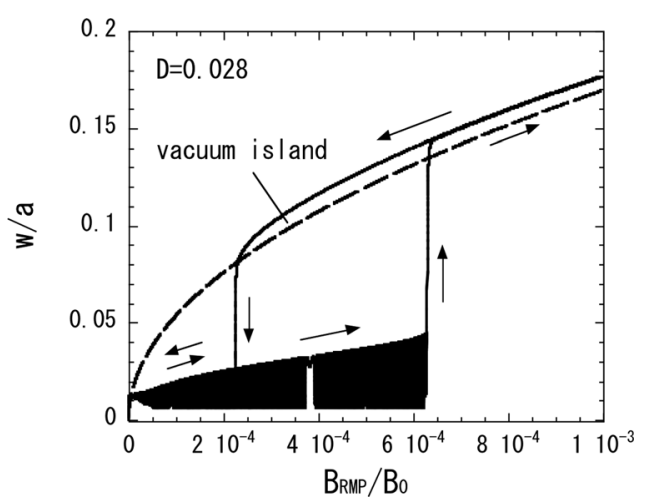

FIG. 3. RMP amplitude dependence of the saturated magnetic island width, where the curvature parameter is $D=0.028$ and the anomalous viscosity coefficient is $\mu=3\left[\mathrm{~m}^{2} / \mathrm{s}\right]$. The dashed line shows the magnetic island width in the vacuum limit.
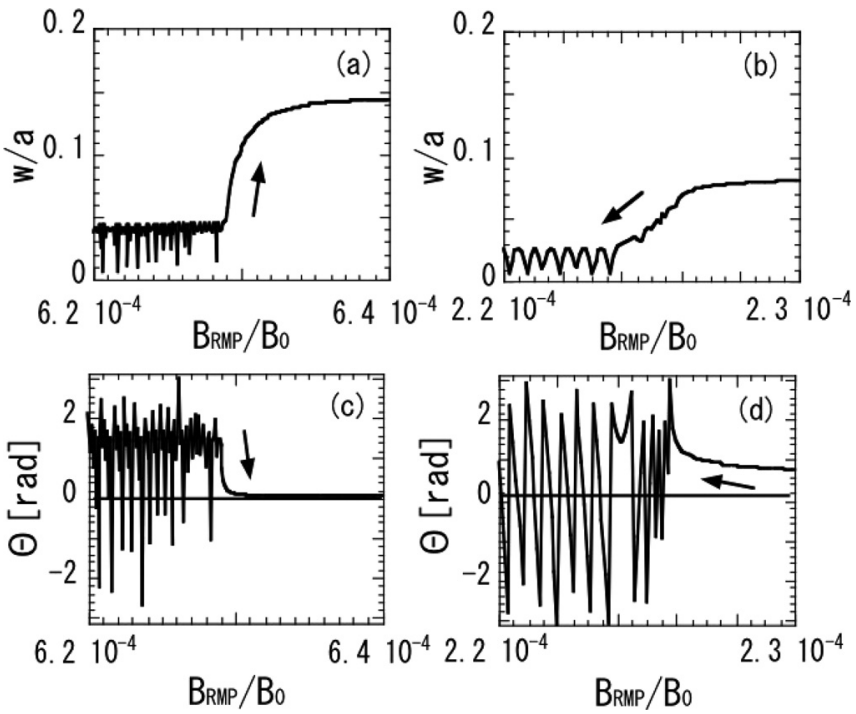

FIG. 4. Extended view of Fig. 4. (a) Island width and (b) island phase in the ramp-up phase, and (c) island width and (d) island phase in the ramp-down phase.

Figure 5 shows the radial profile of poloidal flows near magnetic islands for $D=0.028$ and $\mu=3\left[\mathrm{~m}^{2} / \mathrm{s}\right]$, where the initial island width is given by $w=w_{\mathrm{v}}$. In Fig. 5, to demonstrate the self-healing mechanism by poloidal flows, we amplify the neoclassical velocity in Eq. (110) as

$$
V_{\alpha}^{\text {neo }} \rightarrow f_{V} V_{\alpha}^{\text {neo }}
$$

for $\alpha=\mathrm{i}, \mathrm{e}$, where $f_{V}$ is the amplification factor. In the cases with $f_{V}=0.5,1,1.5$, poloidal flows are damped by the $J \times B$ force near magnetic islands, and the damped poloidal flows and the large locked islands are maintained. While, in the case with $f_{V}=2$, the poloidal flow velocity is large enough so that the viscous force overcomes the $J \times B$ force, and the final state is characterized by unlocked flows and small rotating islands.

Figure 6 shows the stability diagram of magnetic islands in a space of the anomalous viscosity coefficient and the RMP amplitude, where $D=0.028$. It is remarkable that the selfhealing occurs in the small anomalous viscosity limit. In the

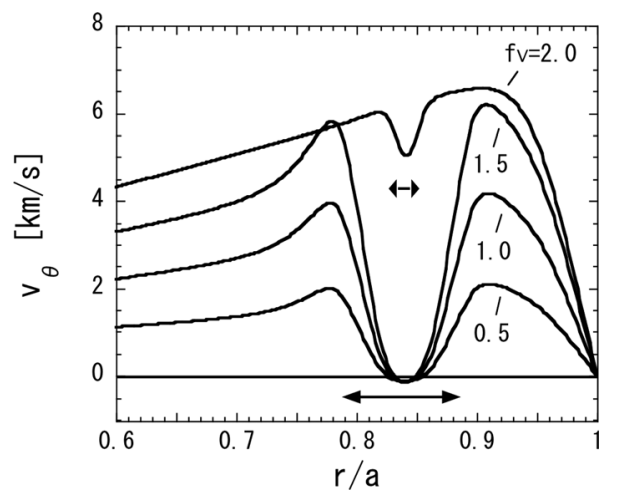

FIG. 5. Radial profile of the poloidal flow velocity near magnetic islands for $D=0.028, \mu=3\left[\mathrm{~m}^{2} / \mathrm{s}\right]$, and $f_{V}=0.5,1,1.5,2.0$, where $f_{V}$ is the amplification factor of the neoclassical flow velocity. Island regions for the locked flows and an unlocked flow are shown by the long and short double-headed arrows, respectively. 


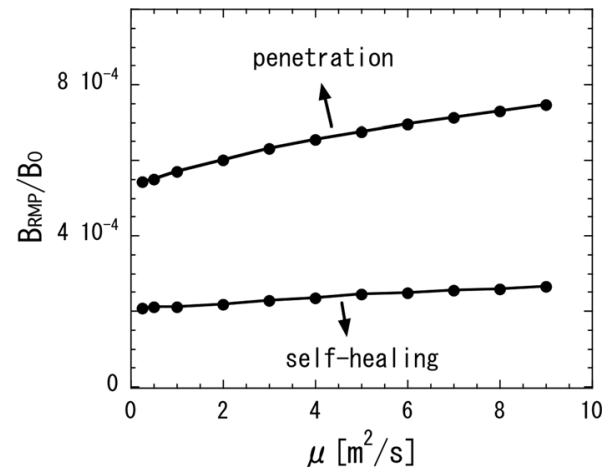

FIG. 6. Stability diagram of magnetic islands in a space of the anomalous viscosity coefficient $\mu$ and the RMP amplitude $B_{\mathrm{RMP}} / B_{0}$, where $D=0.028$.

preceding works, ${ }^{7-9}$ the viscous force is assumed to be driven by the anomalous viscosity coupling the flow gradient just outside the separatrix, which indicates that the self-healing does not occur in the small anomalous viscosity limit. While, in our model, the radial profile of poloidal flows across magnetic islands is taken into account, in consequence, the neoclassical viscosity inside magnetic islands works as a restoring force and triggers the self-healing. This mechanism is consistent with the discussion in Sec. IV, where the self-healing and penetration thresholds in the neoclassical viscosity-dominant regime, Eqs. (127), (139), and (140), are independent from the anomalous viscosity.

To examine the influence of the curvature, we amplify the curvature in Eqs. (53) and (101) as

$$
\kappa_{\mathrm{h}} \rightarrow f_{\kappa} \kappa_{\mathrm{h}},
$$
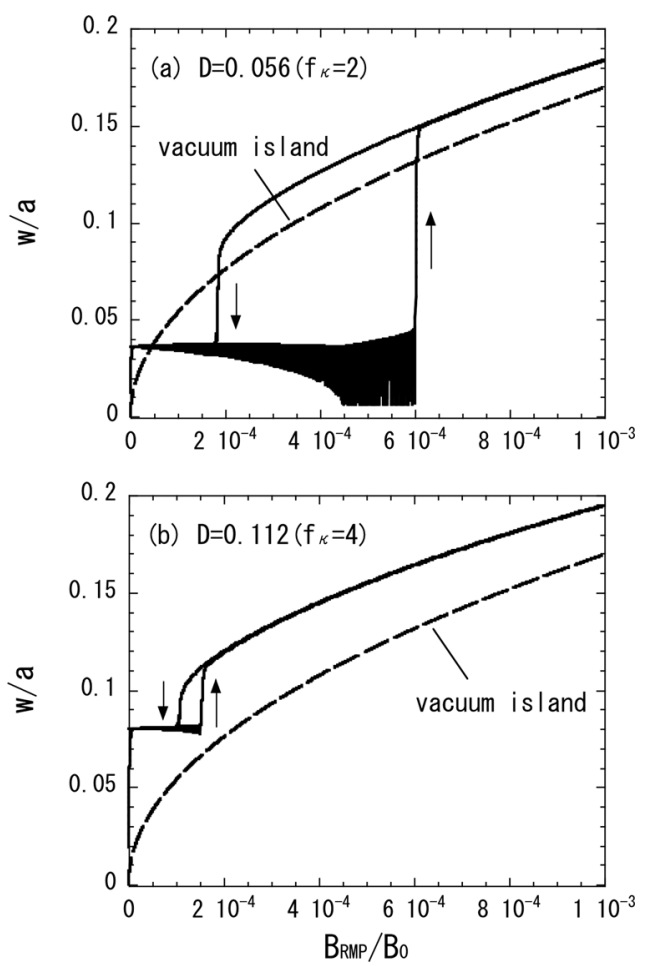

FIG. 7. RMP amplitude dependence of the saturated magnetic island for (a) $D=0.056$ and (b) $D=0.112$, where $\mu=3\left[\mathrm{~m}^{2} / \mathrm{s}\right]$ and $f_{\kappa}$ is the amplification factor of the average curvature.

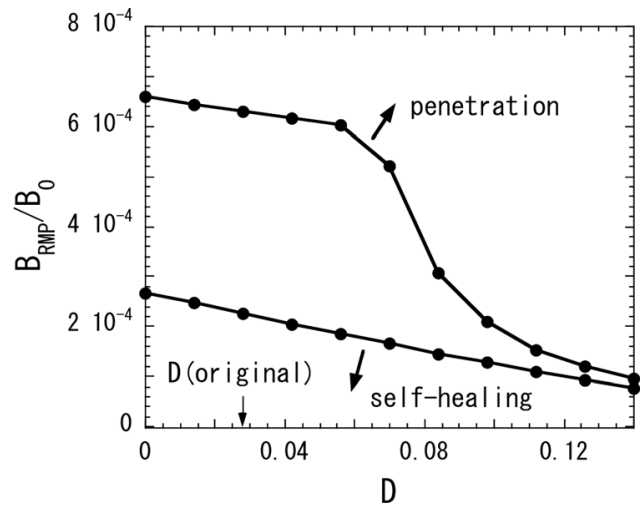

FIG. 8. Stability diagram of magnetic islands in a space of the amplified curvature parameter $D$ and the RMP amplitude, where $\mu=3\left[\mathrm{~m}^{2} / \mathrm{s}\right]$. The original value of $D$ is indicated by an arrow.

where $f_{\kappa}$ is the amplification factor and $f_{\kappa}=0-5$ is considered. The average radial curvature Eq. (36) is given in the limit of the helical symmetry. The operation Eq. (150) mimics the inward and outward shifts of the magnetic axis position and the control of the effective curvature. For $f_{\kappa}=2$ and $f_{\kappa}=4$, the RMP amplitude dependence of the magnetic island width is shown in Fig. 7. In these cases, the curvaturedriven magnetic islands are excited even in the absence of RMP, and the self-healing and penetration thresholds are shifted from that in the case of $f_{\kappa}=1$ in Fig. 3. Figure 8 shows the stability diagram of magnetic islands in a space of the curvature parameter and the RMP amplitude. Figure 8 is similar to Fig. 1 and is consistent with the discussion in Sec. IV, i.e., the self-healing threshold monotonically depends on $D$, while, the penetration threshold weakly depends on $D$ in the small $D$ limit but becomes sensitive to $D$ when the width of the curvature-driven islands overcomes the maximum width of oscillating islands by RMP.

\section{DISCUSSION}

Our model of poloidal flows Eq. (110) is based on the balance between the $J \times B$ force (torque) and the viscous force (torque). The modeling of the $J \times B$ force and the anomalous viscosity are basically the same as those in tokamaks. ${ }^{7-9}$ A different point is that the neoclassical viscosity due to the helical trapped particles is taken into account. ${ }^{24-28}$ In tokamaks, the non-axisymmetric magnetic field perturbation by RMP drives the so-called neoclassical toroidal viscosity. ${ }^{51}$ While, in stellarators, the ambient magnetic field has the considerable non-axisymmetric component $\left(\left|\boldsymbol{B}_{\mathrm{h}}\right| / B_{0} \sim 10^{-1}\right)$, which dominates the influence of RMP. As a result, neoclassical damping rate due to RMP in tokamaks depends on the magnetic island width, while, that in stellarators is independent from the island width but depends on the magnitude of the helical ripple. Therefore, although the force (torque) balance is commonly formulated in both tokamaks and stellarators, the parameter dependence of criteria is different. In this study, the average curvature effect is newly considered, where the effect is stabilizing in tokamaks, while, that is destabilizing in stellarators. Since the average radial curvature typically dominates the average 
toroidal curvature in stellarators, the influence of the curvature is more important than that in tokamaks.

In the following, our results are qualitatively compared with the experimental observations in LHD. The locking (unlocking) of poloidal flows and the excitation (annihilation) of magnetic islands are simultaneously observed, ${ }^{19,22}$ and these facts support that the locking of poloidal flows by RMP-induced $J \times B$ is essential for the island stability. The phase flip of the magnetic diagnostics indicates that magnetic islands start to rotate and shrink at the onset of the selfhealing (Fig. 8 in Ref. 21). According to the theoretical predictions, Eqs. (144) and (146), the critical RMP amplitude for the self-healing shows the weak positive dependence on the $\beta$ value, which is consistent with the experimental results (Fig. 4 in Ref. 20). In the experiments, the self-healing threshold in a parameter space of the $\beta$ value and the normalized collisionality with a fixed RMP amplitude shows monotonic positive dependence (Fig. 8 in Ref. 21), which is also consistent with our results. Our numerical results in a typical parameter regime of LHD show that the magnetic island width at the penetration is of order $10[\mathrm{~cm}]$, which is the value often observed in experiments. Moreover, the island width after the penetration is often larger than the vacuum island width, ${ }^{21}$ which might be explained by the unfavorable curvature effect in our model. All of these similarities might justify our modeling. However, concerning the hysteresis characteristics and the curvature effect, experimental data are not sufficiently accumulated to check our modeling. In addition, an extended analysis with finite toroidal current is necessary to discuss the island bifurcation phenomenon in the low magnetic shear. ${ }^{52}$

\section{SUMMARY}

In this study, a nonlinear theoretical model of magnetic islands and poloidal flows in a sterallator plasma is revisited. We first introduce a fluid model with neoclassical viscosity and effective curvature in stellarators, where the neoclassical viscosity is calculated by radial particle fluxes due to helical trapped particles, and the effective curvature is given by the conventional toroidal averaging method. The asymptotic matching method gives generalized Rutherod equations of the magnetic island width and phase angle in the presence of RMP and the curvature effect. An evolution equation of the poloidal flow across magnetic islands includes the RMPinduced $J \times B$ force, the neoclassical viscosity, and the anomalous viscosity.

Using the model, we obtain criteria of the self-healing of locked magnetic islands and the penetration thresholds of RMP. Scalings of them with dimensionless parameters are also derived. The difference between two criteria gives rise to hysteresis characteristics. In analyses, the poloidal flow profile is categorized into those in the anomalous viscositydominant regime and the neoclassical viscosity-dominant regime. In the former regime, the coupling of anomalous momentum diffusion and the poloidal flow originally excited by the neoclassical viscosity forces locked magnetic islands to rotate and triggers the self-healing. In the latter regime, it is newly found that the self-healing can be driven by the neoclassical viscosity even in the absence of the anomalous viscosity. The self-healing mechanism without the anomalous viscosity is due to the neoclassical viscosity inside magnetic islands. In each regime, the penetration occurs when the RMP amplitude is sufficiently large so that the RMPinduced $J \times B$ force dominates the viscous force. In the presence of the unfavorable curvature, the maximum island width is increased and the criteria of the self-healing and the penetration are modified, where the self-healing tends not to take place, while the penetration tends to occur. The selfhealing threshold is monotonically shifted by the curvature effect. The penetration threshold is less-sensitive to the curvature effect in the small curvature limit, while that becomes sensitive when the curvature-driven tearing mode becomes unstable.

The model is also numerically solved in a typical parameter regime in the LHD. Using ramp-up/down simulations of RMP, the sudden penetration of RMP and the self-healing of magnetic islands are observed, where existence of the hysteresis characteristics is identified. A parameter which characterizes the magnitude of the hysteresis is found. The influence of the curvature effect is checked by increasing effective curvature. The simulation results are consistent with the theoretical prediction.

Finally, difference between our model in stellarators and the standard model in tokamaks is discussed. Our results are also qualitatively compared with the experimental observations in LHD.

In future works, detailed comparison with the experimental observations are necessary. The poloidal flow profile should be analyzed to check the viscosity regime. The influence of the average curvature is desired to be checked, by controlling the magnetic axis position, for example.

\section{ACKNOWLEDGMENTS}

One of the authors, S.N. would like to thank for useful discussion with Dr. Y. Suzuki, Professor K. Itoh, Professor K. Ichiguchi, Dr. A. Ishizawa, Dr. S. Sakakibara, and Professor K. Watanabe. This work was partially supported by Grant-in-Aid for JSPS Fellows (23-5218). We also acknowledge a collaboration program of Research Institute for Applied Mechanics.

${ }^{1}$ F. L. Waelbroeck, Nucl. Fusion 49, 104025 (2009); and references therein. ${ }^{2}$ P. H. Rutherford, Phys. Fluids 16, 1903 (1973).

${ }^{3}$ R. Carrera, R. D. Hazeltine, and M. Kotschenreuther, Phys. Fluids 29, 899 (1986).

${ }^{4}$ M. Kotschenreuther, R. D. Hazeltine, and P. J. Morrison, Phys. Fluids 28, 294 (1985).

${ }^{5}$ A. I. Smolyakov, Plasma Phys. Controlled Fusion 35, 657 (1993).

${ }^{6}$ T. S. Hahm and R. M. Kulsrud, Phys. Fluids 28, 2412 (1985).

${ }^{7}$ R. Fitzpatrick and T. C. Hender, Phys. Fluids B 3, 644 (1991).

${ }^{8}$ R. Fitzpatrick, Nucl. Fusion 33, 1049 (1993).

${ }^{9}$ A. I. Smolyakov, A. Hirose, E. Lazzaro, G. B. Re, and J. D. Callen, Phys. Plasma 2, 1581 (1995).

${ }^{10}$ Y. Kikuchi, M. F. M. de Bock, K. H. Finken, M. Jakubowski, R. Jaspers, H. R. Koslowski, A. Kraemer-Flecken, M. Lehnen, Y. Liang, G. Matsunaga, D. Reiser, R. C. Wolf, O. Zimmermann, and TEXTOR-Team, Phys. Rev. Lett. 97, 085003 (2006)

${ }^{11}$ Q. Yu, S. Günter, Y. Kikuchi, and K. H. Finken, Nucl. Fusion 48, 024007 (2008). 
${ }^{12}$ D. Reiser and D. Chandra, Phys. Plasmas 16, 042317 (2009).

${ }^{13}$ F. Militello and F. L. Waelbroeck, Nucl. Fusion 49, 065018 (2009).

${ }^{14}$ M. Bécoulet, G. Huysmans, X. Garbet, E. Nardon, D. Howell, A. Garofalo, M. Schaffer, T. Evans, K. Shaing, A. Cole, J.-K. Park, and P. Cahyna, Nucl. Fusion 49, 085011 (2009).

${ }^{15}$ E. Nardon, P. Tamain, M. Bécoulet, G. Huysmans, and F. L. Waelbroeck, Nucl. Fusion 50, 034002 (2010).

${ }^{16}$ Y. Liu, A. Kirk, Y. Gribov, M. P. Gryaznevich, T. C. Hender, and E. Nardon, Nucl. Fusion 51, 083002 (2011).

${ }^{17}$ N. M. Ferraro, Phys. Plasmas 19, 056105 (2012).

${ }^{18}$ K. Narihara et al., Phys. Rev. Lett. 87, 135002 (2001).

${ }^{19}$ K. Ida et al., Nucl. Fusion 44, 290 (2004).

${ }^{20}$ N. Ohyabu, Y. Narushima, Y. Nagayama, K. Narihara, T. Morisaki, A. Komori, and LHD Experimental Group, Plasma Phys. Controlled Fusion 47, 1431 (2005).

${ }^{21}$ Y. Narushima, K. Y. Watanabe, S. Sakakibara, K. Narihara, I. Yamada, Y. Suzuki, S. Ohdachi, N. Ohyabu, H. Yamada, Y. Nakamura, and LHD Experimental Group, Nucl. Fusion 48, 075010 (2008).

${ }^{22}$ Y. Narushima, F. Castejón, S. Sakakibara, K. Y. Watanabe, S. Ohdachi, Y. Suzuki, T. Estrada, F. Medina, D. López-Bruna, M. Yokoyama, M. Yoshinuma, K. Ida, S. Nishimura, LHD Experiment Group, and TJ-II Experiment Group, Nucl. Fusion 51, 083030 (2011).

${ }^{23}$ T. Estrada, F. Medina, D. López-Bruna, E. Ascasíbar, R. Balbín, A. Cappa, F. Castejón, S. Eguilior, A. Fernández, J. Guasp, C. Hidalgo, and S. Petrov, Nucl. Fusion 47, 305 (2007).

${ }^{24}$ S. Nishimura, Y. Narushima, S. Toda, M. Yagi, K. Itoh, and S.-I. Itoh, Plasma Fusion Res. 5, 040 (2010).

${ }^{25}$ S. Nishimura, S. Toda, M. Yagi, and Y. Narushima, Plasma Fusion Res. 7, 2403107 (2012).

${ }^{26}$ S. Nishimura, S. Toda, Y. Narushima, and M. Yagi, Plasma Phys. Controlled Fusion 55, 014013 (2013).

${ }^{27}$ C. C. Hegna, Nucl. Fusion 51, 113017 (2011).

${ }^{28}$ C. C. Hegna, Phys. Plasmas 19, 056101 (2012).

${ }^{29}$ R. Fitzpatrick and F. L. Waelbroeck, Phys. Plasmas 19, 112501 (2012).
${ }^{30}$ A. H. Glasser, J. M. Greene, and J. L. Johnson, Phys. Fluids 18, 875 (1975).

${ }^{31}$ J. R. Cary and M. Kotschenreuter, Phys. Fluids 28, 1392 (1985).

${ }^{32}$ C. C. Hegna and A. Bhattacharjee, Phys. Fluids B 1, 392 (1989).

${ }^{33}$ T. Hayashi, T. Sato, and A. Takei, Phys. Fluids B 2, 329 (1990).

${ }^{34}$ Y. Suzuki, N. Nakajima, K. Watanabe, Y. Nakamura, and T. Hayashi, Nucl. Fusion 46, L19 (2006).

${ }^{35}$ R. D. Hazeltine and J. D. Meiss, Plasma Confinement (Dover, New York, 2003).

${ }^{36}$ R. D. Hazeltine, M. Kotschenreuter, and P. J. Morrison, Phys. Fluids 28, 2466 (1985).

${ }^{37}$ S. I. Braginskii, Reviews of Plasma Physics (Consultants Bureau, New York, 1965), Vol. 1, p. 205.

${ }^{38}$ C. T. Hsu, R. D. Hazeltine, and P. J. Morrison, Phys. Fluids 29, 1480 (1986)

${ }^{39}$ M. Yagi, "Study of anomalous transport based on drift and resistive instabilities in heliotron/torsatron configuration," Ph.D. dissertation, Kyoto University (1989).

${ }^{40}$ Z. Chang and J. D. Callen, Phys. Fluids B 4, 1766 (1992).

${ }^{41}$ T. Tala et al., Plasma Phys. Controlled Fusion 49, B291 (2007).

${ }^{42}$ A. G. Peeters et al., Nucl. Fusion 51, 094027 (2011); and references therein.

${ }^{43}$ A. Ishizawa and N. Nakajima, Phys. Plasmas 17, 072308 (2010).

${ }^{44}$ H. R. Strauss, Plasma Phys. 22, 733 (1980).

${ }^{45} \mathrm{M}$. Wakatani, Stellarator and Heliotron Devices (Oxford University Press, New York, 1998).

${ }^{46}$ K. C. Shaing, Phys. Fluids 27, 1567 (1984).

${ }^{47}$ R. Fitzpatrick, Phys. Plasmas 2, 825 (1995).

${ }^{48}$ H. Lütjens, J.-F. Luciani, and X. Garbet, Phys. Plasmas 8, 4267 (2001).

${ }^{49}$ A. Pytte and A. H. Boozer, Phys. Fluids 24, 88 (1981).

${ }^{50}$ S. Toda, K. Itoh, A. Fujisawa, S.-I. Itoh, M. Yagi, A. Fukuyama, P. H. Diamond, and K. Ida, Nucl. Fusion 47, 914 (2007).

${ }^{51}$ J. D. Callen, Nucl. Fusion 51, 094026 (2011); and references therein.

${ }^{52}$ K. Ida, S. Inagaki, M. Yoshinuma, Y. Narushima, K. Itoh, T. Kobuchi, K. Y. Watanabe, H. Funaba, S. Sakakibara, T. Morisaki, and LHD Experimental Group, Phys. Rev. Lett. 100, 045003 (2008). 